\title{
Magnetic interactions in a series of homodinuclear lanthanide complexes
}

Peter Comba, ${ }^{1, *}$ Michael Großhauser, ${ }^{1}$ Rüdiger Klingeler, ${ }^{2}$ Changhyun Koo, ${ }^{2}$ Yanhua Lan, ${ }^{3}$ Dennis Müller, ${ }^{1}$ Jaena Park, ${ }^{2}$ Annie Powell, ${ }^{3}$ Mark J. Riley, ${ }^{4}$ and Hubert Wadepohl. ${ }^{1}$

${ }^{1}$ Universität Heidelberg, Anorganisch-Chemisches Institut und Interdisziplinäres Zentrum für Wissenschaftliches Rechnen (IWR), INF 270,

D-69120 Heidelberg, Germany

${ }^{2}$ Universität Heidelberg, Kirchhoff-Institut für Physik, INF 227, D-69120 Heidelberg, Germany

${ }^{3}$ Institut für Anorganische Chemie, Karlsruhe Institute of Technology (KIT), Engesserstr. 15, Geb. 30.45, D-76131 Karlsruhe, and Institute of Nanotechnology (INT), P.O. Box 3640, D76021, Karlsruhe, Germany

${ }^{4}$ University of Queensland, School of Chemistry and Molecular Biosciences, Brisbane, QLD 4072, Australia

\section{Supporting Information}

Correspondence:

Fax: +49-6221-546617

E-mail: peter.comba@aci.uni-heidelberg.de 
Table S1. Crystal data of the homodinuclear $\operatorname{Ln}{ }^{\prime \prime \prime}{ }_{2}$ complexes.

\begin{tabular}{|c|c|c|c|c|c|c|c|}
\hline & {$\left[Y_{2} L(O A c)_{4}\right] P F_{6}$} & {$\left[G d_{2} L(O A c)_{4}\right] P F_{6}$} & {$\left[T b_{2} L(O A c)_{4}\right] P F_{6}$} & {$\left[D y_{2} L(O A c)_{4}\right] P F_{6}$} & {$\left[\mathrm{Ho}_{2} \mathrm{~L}(\mathrm{OAc})_{4}\right] \mathrm{PF}_{6}$} & {$\left[E r_{2} L(O A c)_{4}\right] P F_{6}$} & {$\left[L u_{2} L(O A c)_{4}\right] P F_{6}$} \\
\hline Empirical formula & $\mathrm{C}_{44} \mathrm{H}_{57} \mathrm{~F}_{6} \mathrm{~N}_{6} \mathrm{O}_{12} \mathrm{PY}_{2}$ & $\mathrm{C}_{44} \mathrm{H}_{57} \mathrm{~F}_{6} \mathrm{Gd}_{2} \mathrm{~N}_{6} \mathrm{O}_{12} \mathrm{P}$ & $\mathrm{C}_{44} \mathrm{H}_{57} \mathrm{~F}_{6} \mathrm{~N}_{6} \mathrm{O}_{12} \mathrm{PTb}_{2}$ & $\mathrm{C}_{41} \mathrm{H}_{45} \mathrm{Dy}_{2} \mathrm{~F}_{6} \mathrm{~N}_{6} \mathrm{O}_{9} \mathrm{P}$ & $\mathrm{C}_{44} \mathrm{H}_{57} \mathrm{~F}_{6} \mathrm{Ho}_{2} \mathrm{~N}_{6} \mathrm{O}_{12} \mathrm{P}$ & $\mathrm{C}_{41} \mathrm{H}_{45} \mathrm{Er}_{2} \mathrm{~F}_{6} \mathrm{~N}_{6} \mathrm{O}_{9} \mathrm{P}$ & $\mathrm{C}_{44} \mathrm{H}_{57} \mathrm{~F}_{6} \mathrm{Lu}_{2} \mathrm{~N}_{6} \mathrm{O}_{12} \mathrm{P}$ \\
\hline Formula weight & 1184.74 & 1321.42 & 1324.76 & 1235.80 & 1336.78 & 1245.32 & 1356.86 \\
\hline$T / K$ & $100(1)$ & $100(1)$ & $100(1)$ & $100(1)$ & $100(1)$ & $110(1)$ & $110(1)$ \\
\hline Crystal system & Monoclinic & Monoclinic & Monoclinic & Monoclinic & Monoclinic & Monoclinic & Monoclinic \\
\hline Space group & $\mathrm{P} 21 / \mathrm{c}$ & $\mathrm{P} 21 / \mathrm{c}$ & $\mathrm{P} 21 / \mathrm{c}$ & $\mathrm{P} 21 / \mathrm{c}$ & P $21 / c$ & $\mathrm{P} 21 / \mathrm{c}$ & $\mathrm{P} 21 / \mathrm{c}$ \\
\hline Unit cell dimensions a/ $A$ & $14.032(11)$ & $14.04396(10)$ & $14.039(7)$ & $14.014(6)$ & $13.970(9)$ & $13.98350(8)$ & $14.04396(10)$ \\
\hline$b / \AA$ & $17.572(14)$ & $17.51192(9)$ & $17.556(10)$ & $17.576(7)$ & $17.521(12)$ & $17.51219(9)$ & $17.51192(9)$ \\
\hline$c / \AA$ & $20.453(14)$ & $20.36011(10)$ & $20.505(9)$ & $20.496(8)$ & $20.414(13)$ & $20.49149(10)$ & $20.36011(10$ \\
\hline$\alpha / \operatorname{deg}$ & $90^{\circ}$ & $90^{\circ}$ & $90^{\circ}$ & $90^{\circ}$ & $90^{\circ}$ & $90^{\circ}$ & $90^{\circ}$ \\
\hline$\beta / \operatorname{deg}$ & $99.119(9)^{\circ}$ & $98.7154(6)^{\circ}$ & $99.310(12)^{\circ}$ & $99.160(12)^{\circ}$ & $99.119(8)^{\circ}$ & $99.0144(5)^{\circ}$ & $98.7154(6)^{\circ}$ \\
\hline$\gamma / \mathrm{deg}$ & $90^{\circ}$ & $90^{\circ}$ & $90^{\circ}$ & $90^{\circ}$ & $90^{\circ}$ & $90^{\circ}$ & $90^{\circ}$ \\
\hline Volume/ $A^{3}$ & 4979(7) & $4949.48(5)$ & $4987(4)$ & 4984(4) & $4934(6)$ & $4956.01(4)$ & $4949.48(5)$ \\
\hline$Z$ & 4 & 4 & 4 & 4 & 4 & 4 & 4 \\
\hline density (calc.) $/ M g \cdot m^{-3}$ & 1.580 & 1.773 & 1.764 & 1.647 & 1.800 & 1.669 & 1.821 \\
\hline Absorption coefficient / $\mathrm{mm}^{-1}$ & 2.439 & 2.778 & 2.933 & 3.084 & 3.305 & 3.473 & 4.087 \\
\hline$F_{000}$ & 2424 & 2624 & 2632 & 2424 & 2648 & 2440 & 2680 \\
\hline Crystal size / $\mathrm{mm}^{3}$ & 0.4 to 0.1 to 0.01 & 0.4 to 0.1 to 0.01 & 0.4 to 0.1 to 0.01 & 0.26 to 0.18 to 0.09 & 0.4 to 0.1 to 0.01 & $\begin{array}{l}0.2047 \text { to } 0.1230 \text { to } \\
0.0563\end{array}$ & $\begin{array}{l}0.2617 \text { to } 0.2275 \text { to } \\
0.0223\end{array}$ \\
\hline Independent reflections $\left[R_{\text {int }}\right]$ & $8829[0.1286]$ & $17448[0.0412]$ & $17249[0.0920]$ & $17183[0.0420]$ & $15144[0.0848]$ & $19909[0.0506]$ & $20139[0.1074]$ \\
\hline Observed reflections $[I>2 \sigma(I)]$ & 5608 & 15091 & 12327 & 14634 & 11576 & 17447 & 15873 \\
\hline Index ranges $h, k, l$ & $\begin{array}{l}-16 \text { to } 16,-20 \text { to } 20,-24 \\
\text { to } 24\end{array}$ & $\begin{array}{l}-20 \text { to } 21,-26 \text { to } 26,-30 \\
\text { to } 30\end{array}$ & $\begin{array}{l}-21 \text { to } 21,-26 \text { to } 26,-30 \\
\text { to } 30\end{array}$ & $\begin{array}{l}-21 \text { to } 20,-25 \text { to } 26,-30 \\
\text { to } 30\end{array}$ & $\begin{array}{l}-19 \text { to } 19,-24 \text { to } 25,-29 \\
\text { to } 29\end{array}$ & $\begin{array}{l}-22 \text { to } 22,-27 \text { to } 27,-31 \\
\text { to } 32\end{array}$ & $\begin{array}{l}-22 \text { to } 22,-27 \text { to } 27,-32 \\
\text { to } 32\end{array}$ \\
\hline Transmission factors: max, min & $0.7452,0.5508$ & $0.4348,0.3561$ & $0.4348,0.3460$ & $0.9304,0.5878$ & $1.0000,0.7349$ & $0.841,0.567$ & $1.000,0.254$ \\
\hline Data / restraints / parameters & $8829 / 160 / 648$ & $17448 / 120 / 591$ & $17249 / 120 / 590$ & $17183 / 0 / 591$ & $15144 / 120 / 591$ & $19909 / 120 / 591$ & $20139 / 160 / 648$ \\
\hline Goodness-of-fit on $F^{2}$ & 1.045 & 1.097 & 1.028 & 1.057 & 1.046 & 1.056 & 1.045 \\
\hline $\begin{array}{l}\text { Final R indices }\left[F_{0}>4 \sigma\left(F_{0}\right)\right] R(F) \text {, } \\
w R\left(F^{2}\right)\end{array}$ & $0.0536,0.1264$ & $0.0244,0.0639$ & $0.0415,0.0988$ & $0.0254,0.0557$ & $0.0348,0.0886$ & $0.0282,0.0667$ & $0.0365,0.0780$ \\
\hline $\begin{array}{l}\text { Final R indices (all data) } R(F) \text {, } \\
w R\left(F^{2}\right)\end{array}$ & $0.1084,0.1535$ & $0.0324,0.0714$ & $0.0755,0.1144$ & $0.0360,0.0618$ & $0.0559,0.1003$ & $0.0351,0.0701$ & $0.0557,0.0856$ \\
\hline Difference density: rms, $\max , \min$ & $0.146,0.869,-1.127$ & $0.178,1.421,-1.085$ & $0.235,1.575,-1.431$ & $0.138,1.881,-0.960$ & $0.220,2.202,-2.303$ & $0.136,2.630,-0.993$ & $0.169,2.425,-1.343$ \\
\hline
\end{tabular}




\section{X-ray Crystal Structure Determinations}

Crystal data and details of the structure determinations are listed in Table S1. Full shells of intensity data were collected at low temperature using Mo- $K_{\alpha}$ radiation $(\lambda=0.71073 \AA$ ) with a Agilent Technologies Supernova-E CCD diffractometer (microfocus tube, multilayer mirror optics; complexes with Ln $=$ Er, Lu) or with a Bruker AXS Smart 1000 CCD diffractometer (sealed tube, graphite monochromator; all other complexes). Data were corrected for air and detector absorption, Lorentz and polarization effects; ${ }^{1,2}$ absorption by the crystal was treated numerically (Gaussian grid, complexes with $\mathrm{Ln}=\mathrm{Er}, \mathrm{Lu})^{2,3}$ or with a semiempirical multiscan method (all other complexes)..$^{3-5}$ The structures were solved by the charge flip procedure $^{6}$ and refined by full-matrix least squares methods based on $F^{2}$ against all unique reflections. ${ }^{7-9}$ All non-hydrogen atoms were given anisotropic displacement parameters. Hydrogen atoms were input at calculated positions and refined with a riding model. All crystals contained solvent of crystallization (methanol) which was frequently disordered and, with appropriate geometry and adp restraints, could be included in the refinement only in two cases $(\mathrm{Ln}=\mathrm{Y}$ and $\mathrm{Lu})$. In all other cases, the electron density attributed to solvent of crystallization was removed from the structures with the BYPASS procedure, ${ }^{10}$ as implemented in PLATON (SQUEEZE). ${ }^{11,} 12$ Partial structure factors from the solvent masks were included in the refinement as separate contributions to $F_{\text {obs. }}$. When found necessary the hexafluorophosphate anions were subjected to geometry restraints during refinement to give sensible P-F and F $\cdots F$ distances. 
Figure S1. ORTEP plots of the complexes
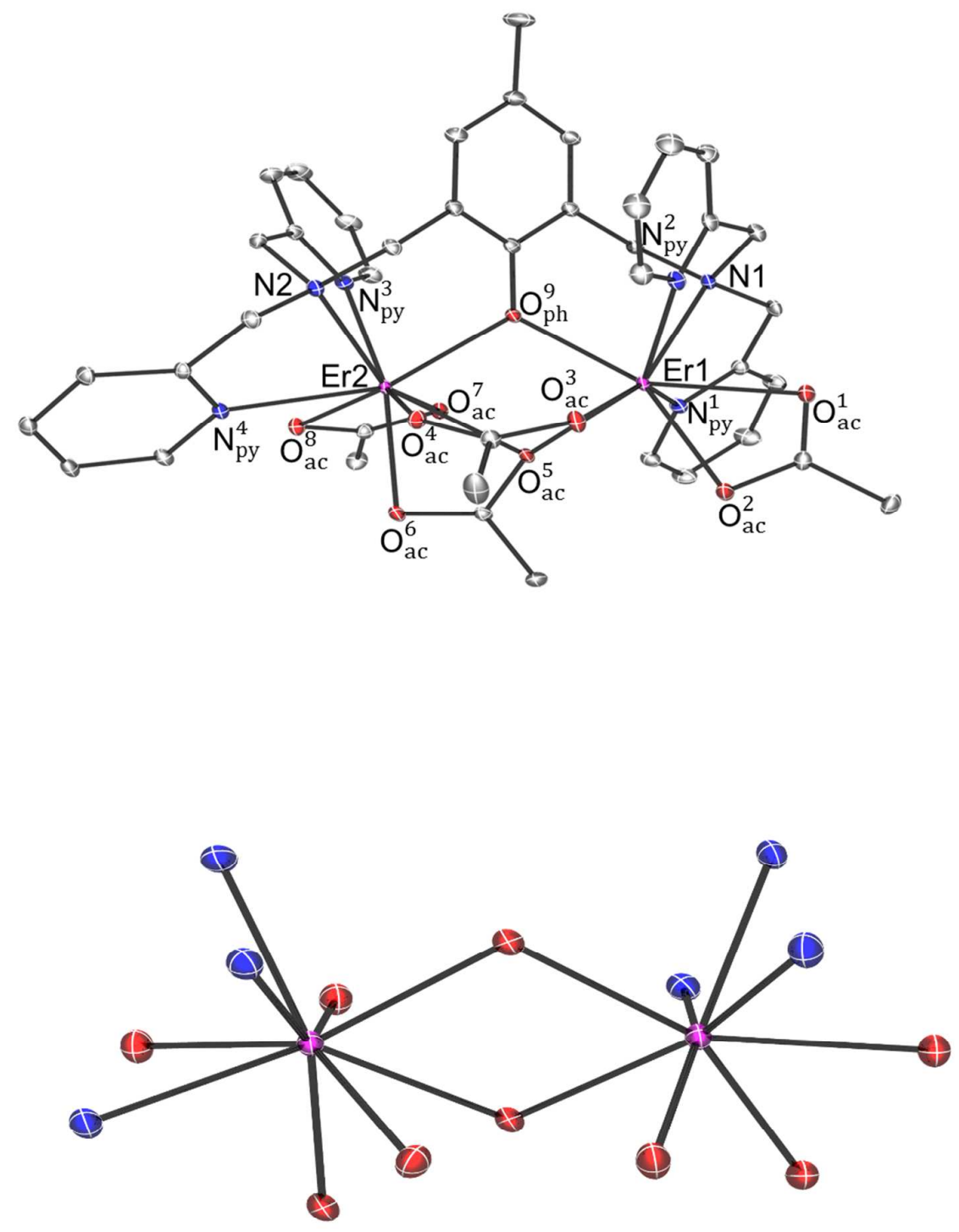

$\mathrm{Er}^{\mathrm{III}}{ }_{2}$ 

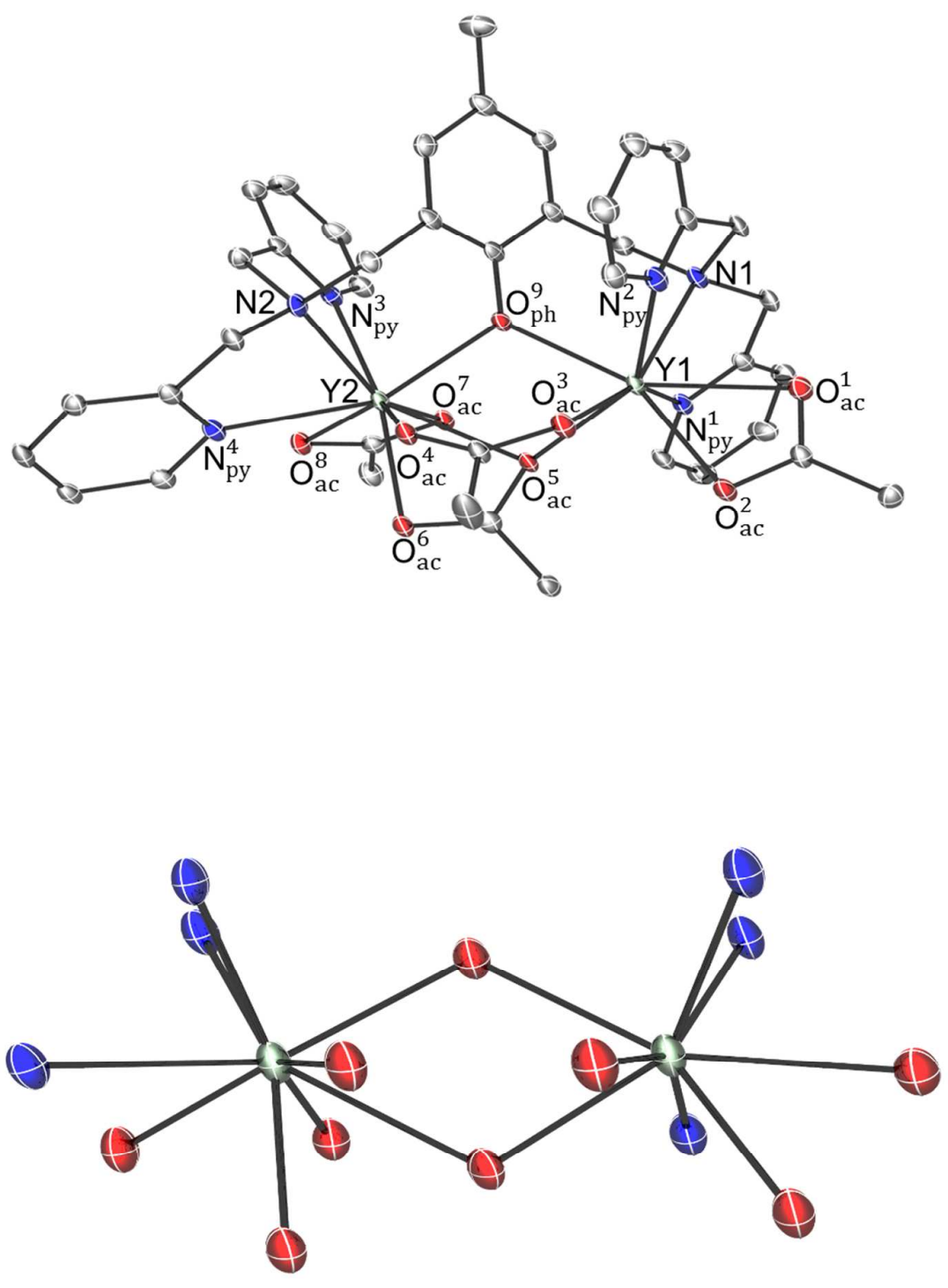

$\mathrm{Y}^{\mathrm{III}}{ }_{2}$ 

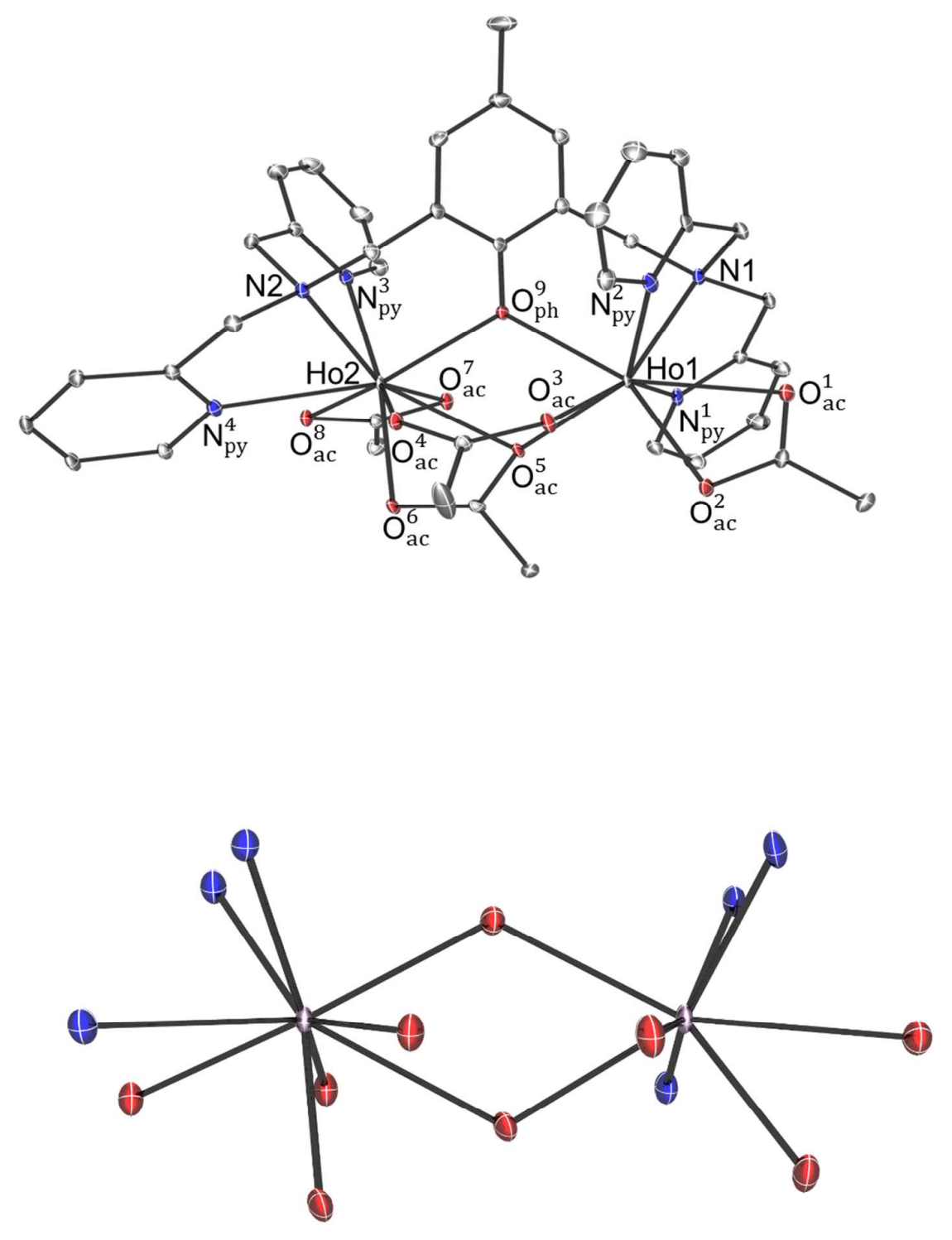

$\mathrm{Ho}_{2}{ }_{2}$ 

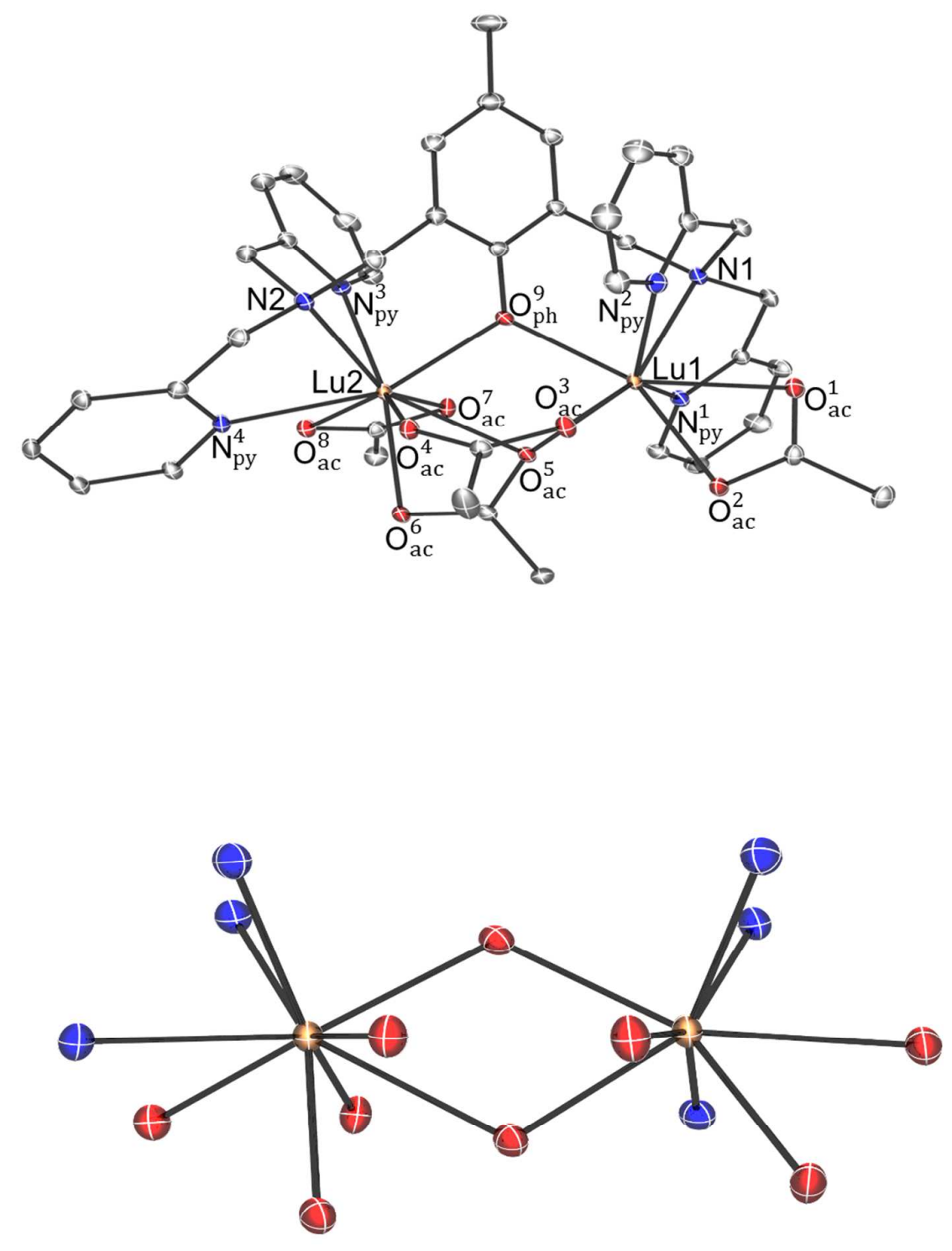

$\mathrm{Lu}_{2}{ }_{2}$ 

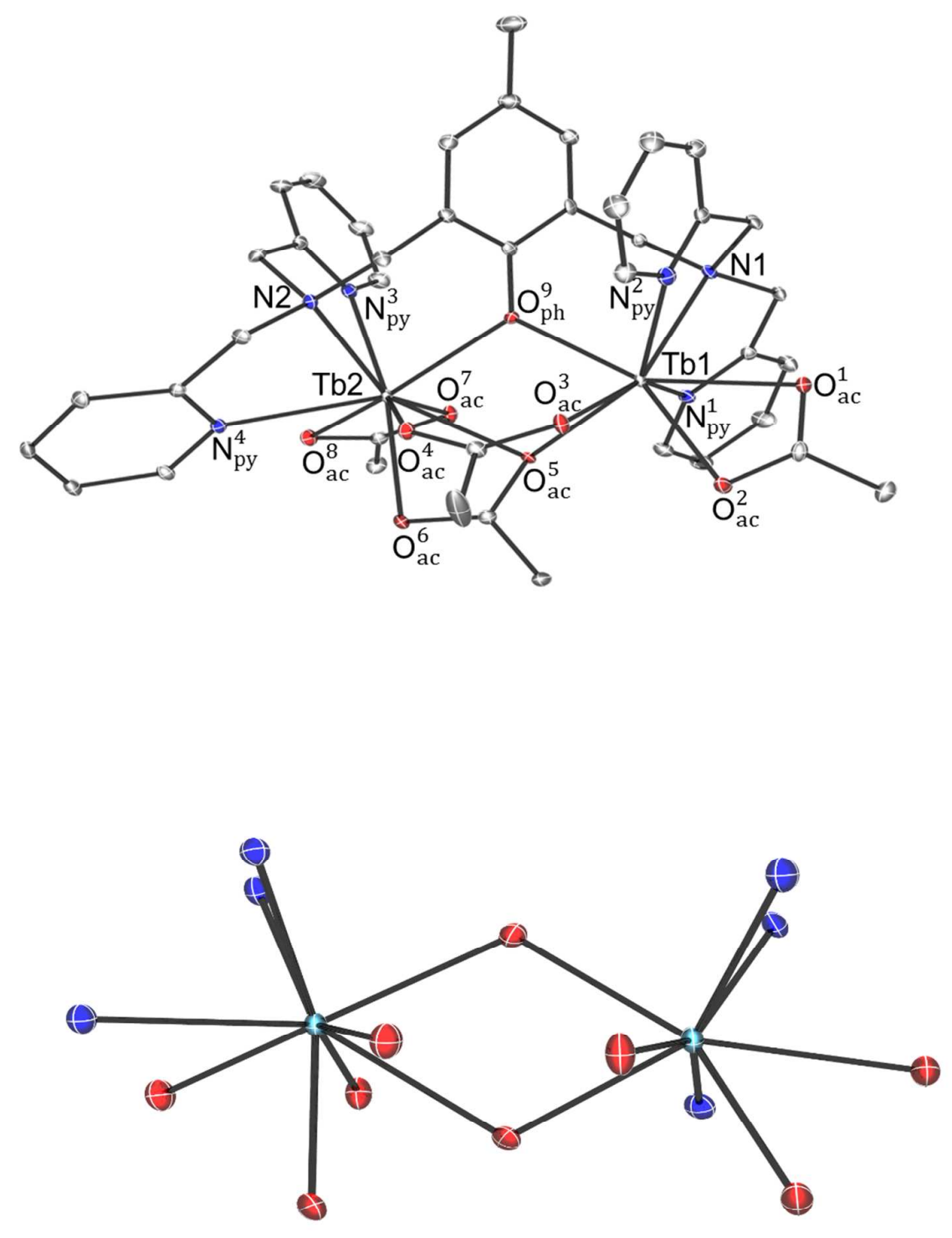

$\mathrm{Tb}_{2}{ }_{2}$ 

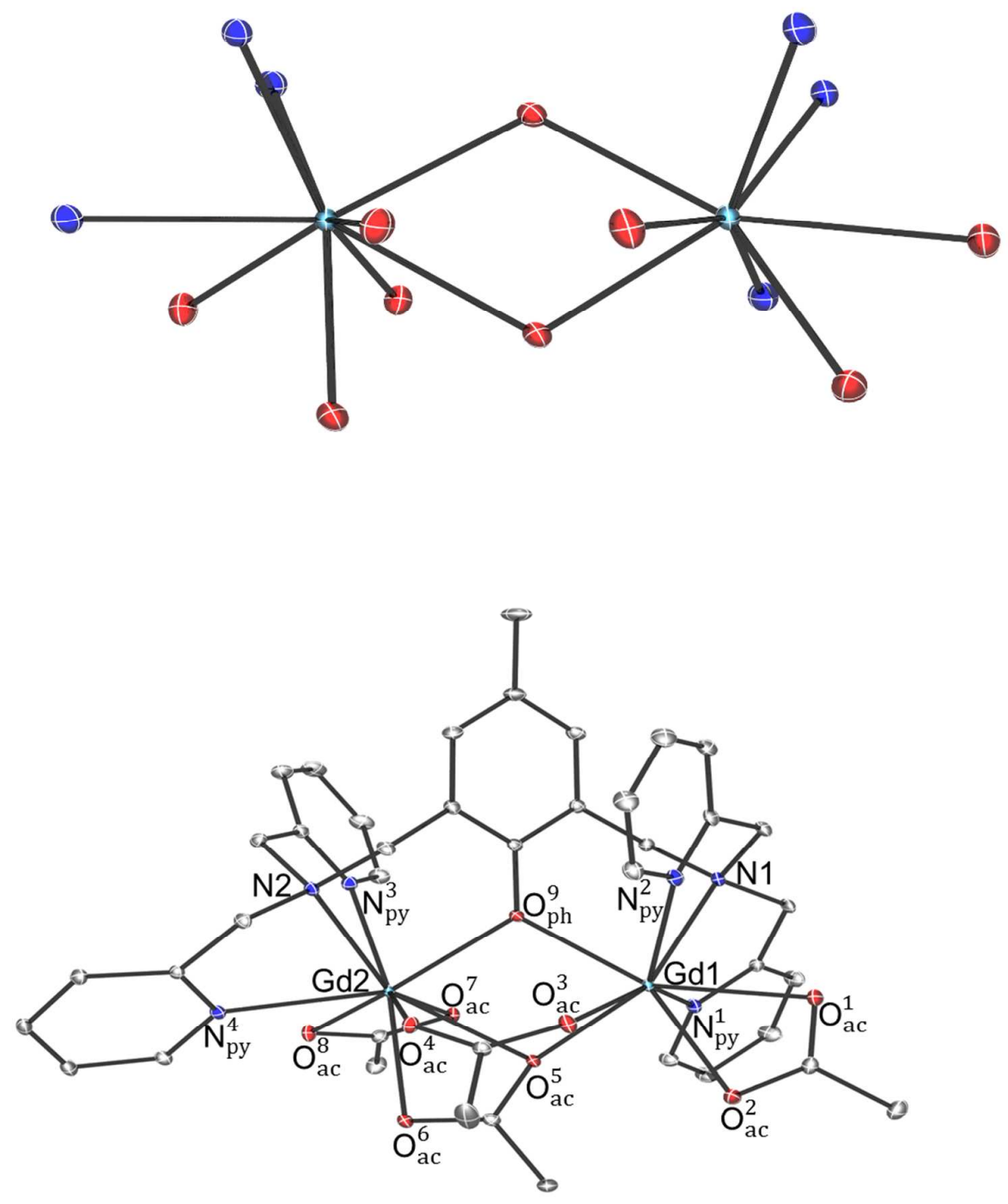

$\mathrm{Gd}^{\mathrm{III}}{ }_{2}$ 

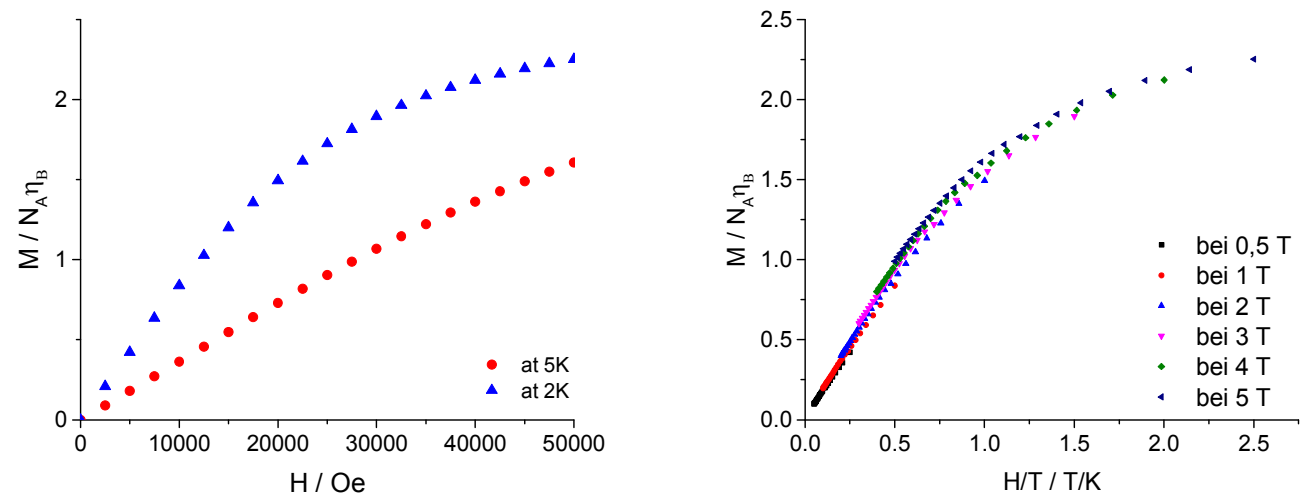

Figure S2. Plots of the magnetization vs. field $H$ (left) and magnetization vs. $H / T$ (right) of $\left[\mathrm{Nd}_{2} \mathrm{~L}(\mathrm{OAc})_{4}\right] \mathrm{PF}_{6}$.
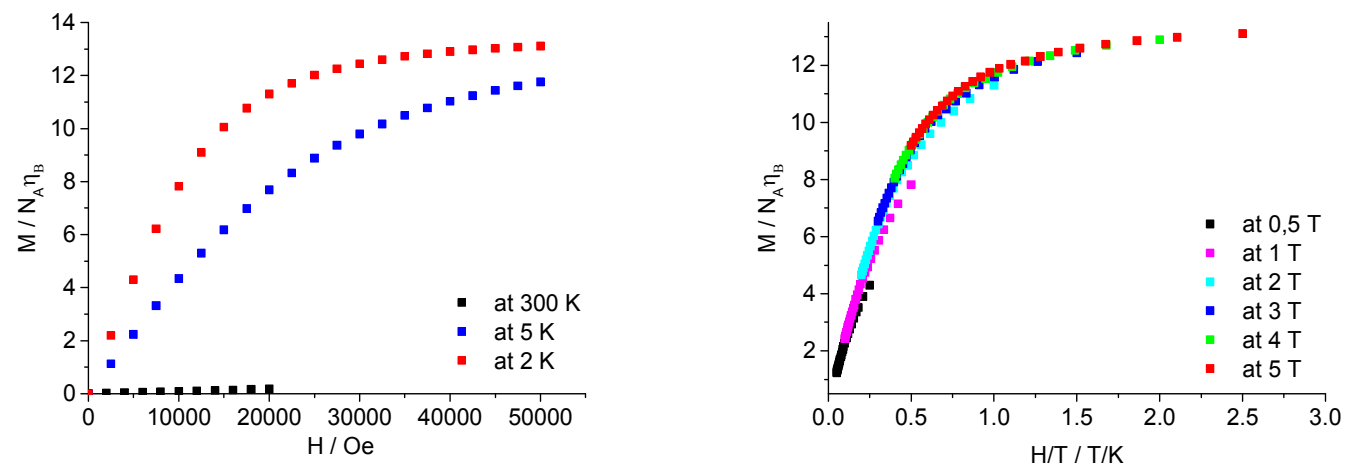

Figure S3. Plots for the Magnetization vs. $H$ (left) and Magnetization vs. H/T (right) for $\left[\mathrm{Gd}_{2} \mathrm{~L}(\mathrm{OAC})_{4}\right] \mathrm{PF}_{6}$. 

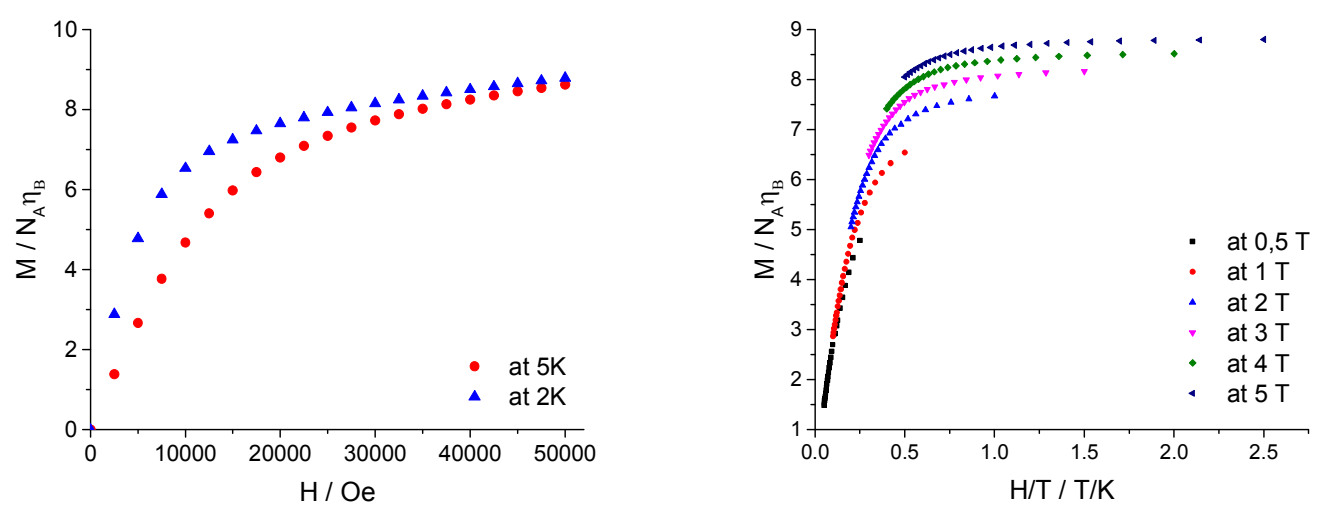

Figure S4. Plots for the Magnetization vs. $\mathrm{H}$ (left) and Magnetization vs. H/T (right) for $\left[\mathrm{Tb}_{2} \mathrm{~L}(\mathrm{OAC})_{4}\right] \mathrm{PF}_{6}$.
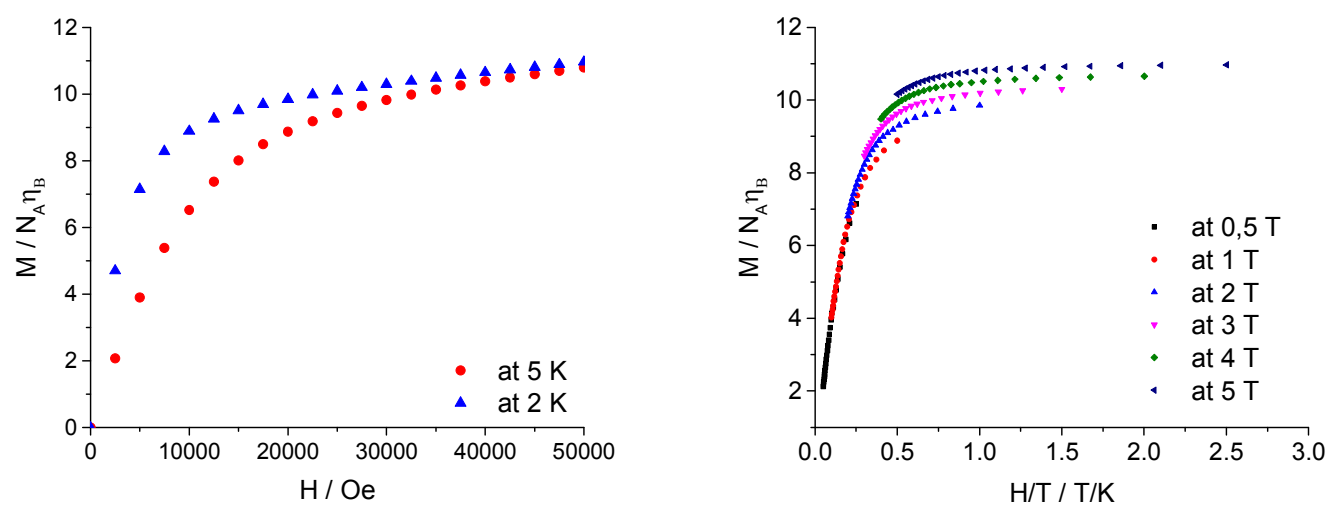

Figure S5. Plots for the Magnetization vs. $\mathrm{H}$ (left) and Magnetization vs. H/T (right) for $\left[\mathrm{Dy}_{2} \mathrm{~L}(\mathrm{OAc})_{4}\right] \mathrm{PF}_{6}$. 

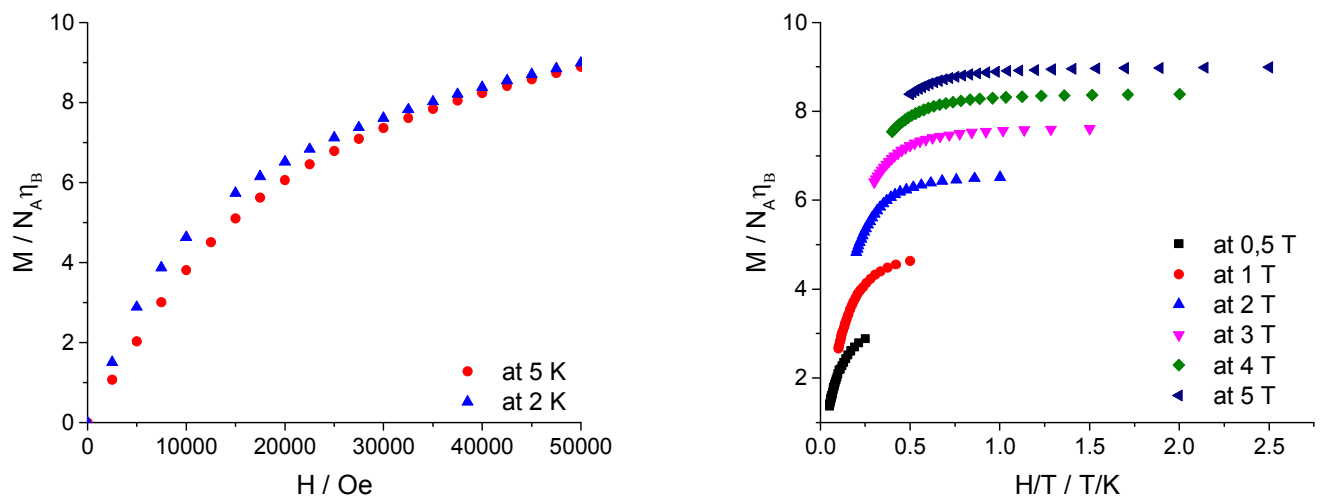

Figure S6. Plots for the Magnetization vs. $\mathrm{H}$ (left) and Magnetization vs. $H / T$ (right) for $\left[\mathrm{Ho}_{2} \mathrm{~L}(\mathrm{OAC})_{4}\right] \mathrm{PF}_{6}$.
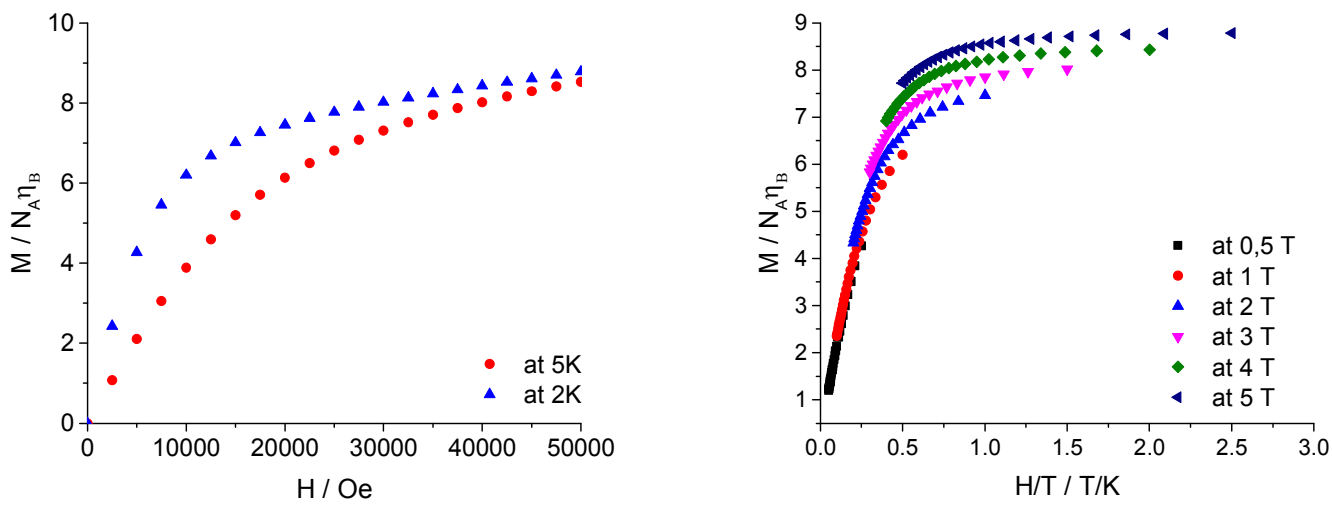

Figure S7. Plots for the Magnetization vs. $H$ (left) and Magnetization vs. $H / T$ (right) for $\left[\mathrm{Nd}_{2} \mathrm{~L}(\mathrm{OAc})_{4}\right] \mathrm{PF}_{6}$. 
(a)

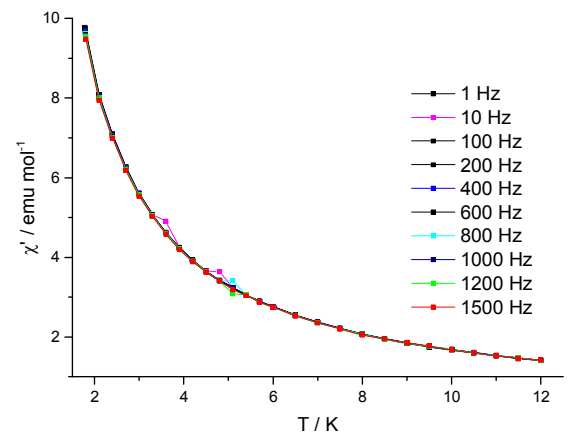

(b)

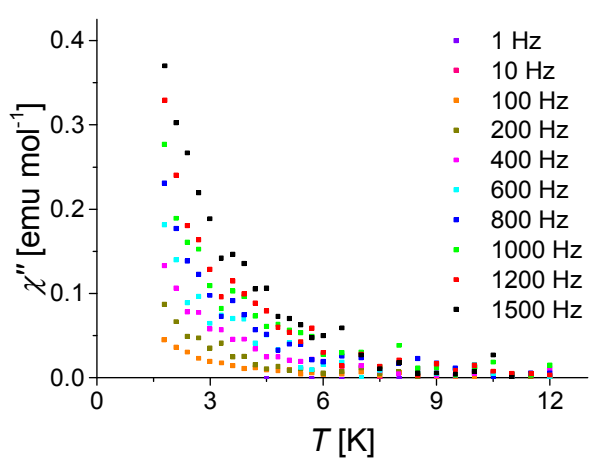

(c)

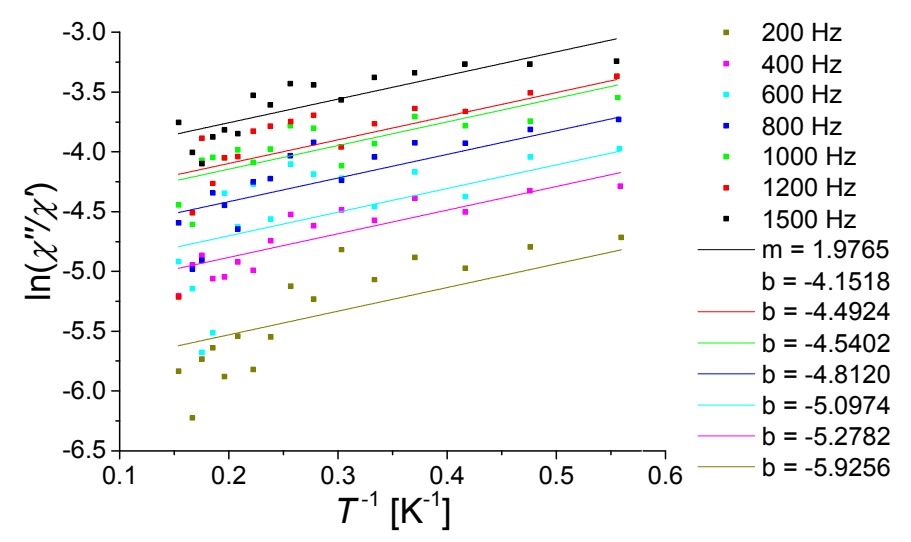

Figure S8. Temperature dependence of the in-phase (a) and out-phase (b) AC susceptibility of $\left[\mathrm{Dy}^{\mathrm{III}}{ }_{2}(\mathrm{~L})(\mathrm{OAC})_{4}\right] \mathrm{PF}_{6}$ at zero $\mathrm{DC}$ field, and (c) plot of the natural logarithm of the ratio of $\chi^{\prime \prime} / \chi^{\prime}$ as a function of $T^{1}$ of $\left[\mathrm{Dy}^{\prime \prime \prime}{ }_{2}(\mathrm{~L})(\mathrm{OAC})_{4}\right] \mathrm{PF}_{6}$. The slope corresponds to $U_{\text {eff }}=1.97 \mathrm{~K}$. 


\section{Spectroscopy}

If the ligand field is defined by Görller-Walrand and Binnemans is given by

$$
\mathrm{H}_{\mathrm{LF}}=\sum_{k=2,4,6} B_{k 0} C_{0}^{k}+\sum_{q=1}^{k}\left[B_{k q}\left(C_{-q}^{k}+(-1)^{q} C_{q}^{k}\right)+i B_{k q}^{\prime}\left(C_{-q}^{k}-(-1)^{q} C_{q}^{k}\right)\right]
$$

where the tensor operators $C_{q}^{k}$ are related to the spherical harmonics $Y_{q}^{k}$ by $C_{q}^{k}=\sqrt{\frac{4 \pi}{2 k+1}} Y_{q}^{k}$. Although the ligand field can be complex, the 27 possible $B_{k q}, B_{k q}{ }^{\prime}$ parameters are all real. The matrix given in Table 3 of Urland [16] relates the ligand field defined as the complex coefficients $\left(\mathrm{R}_{\mathrm{k}} \mathrm{c}_{\mathrm{kq}}\right)$ of $Y_{q}^{k}$ to the 28 real matrix elements $\left\langle\mathrm{f}\left|\mathrm{V}_{\mathrm{LF}}\right| \mathrm{f}^{\prime}\right\rangle$ of the upper triangle of the $7 \times 7 \mathrm{AOM}$ matrix in the basis of real f-orbitals (note that the coefficient $\bar{E}$ in Table 3 should be $\frac{2}{5} \sqrt{\frac{52 \pi}{7}}$.[13]) The AOM matrix itself has been given Table 2 in terms of the Euler angles $\theta, \varphi$ and the AOM parameters $\mathrm{e}_{\sigma}, \mathrm{e}_{\pi}$. This is analogous to the well-known AOM matrix for d-orbitals given, for example, by Schaffer.[14] Here we assume that the AOM parameters are isotropic $\left(\mathrm{e}_{\pi \mathrm{x}}=\mathrm{e}_{\pi \mathrm{y}}\right)$.

The Table 3 of Urland [16] reduces to a $27 \times 27$ real matrix as the trace of the AOM matrix only contributes to an overall shift of the levels (corresponding to $B_{00}$ ) and can be ignored, and the $B_{k q}$ parameters of Equation (A1.1) are related to the $R_{k} c_{k q}$ given in Table 3 by

$$
B_{k q}=(-1)^{\mathrm{q}} \sqrt{\frac{2 \mathrm{k}+1}{4 \pi}} \operatorname{Re}\left(\mathrm{R}_{\mathrm{k}} \mathrm{c}_{\mathrm{kq}}\right) ; \quad B_{k q}{ }^{\prime}=-(-1)^{\mathrm{q}} \sqrt{\frac{2 \mathrm{k}+1}{4 \pi}} \operatorname{Im}\left(\mathrm{R}_{\mathrm{k}} \mathrm{c}_{\mathrm{kq}}\right)
$$

Using this approach, a ligand field can be estimated from the angular position of the ligands and a reasonable estimate of the AOM parameters. A detailed study of a mixed $\mathrm{N}, \mathrm{O}$ coordinating ligand has been made for nine lanthanide complexes and the $B_{k q}$ ligand field parameters have been fitted to their spectra.[15] A single set of $e_{\sigma}, e_{\pi}$ parameters could be found the ligands that could reproduce the $B_{k q}$ parameters when adjusted for changing geometry and bond lengths. The values found were $e_{\sigma}=384 \mathrm{~cm}^{-1}, \mathrm{e}_{\pi}=0$ for a tertiary amine $(R=2.739 \AA) ; e_{\sigma}=742 \mathrm{~cm}^{-1}, e_{\pi}=122 \mathrm{~cm}^{-1}$ for an imine $(R=2.491 \AA) ;$ and $e_{\sigma}=258 \mathrm{~cm}^{-1}, \mathrm{e}_{\pi}=190$ $\mathrm{cm}^{-1}$ for a phenolate $(R=2.222 \AA)$.[15] When these values are scaled for the bond lengths in the $\left[\mathrm{Ln}_{2} \mathrm{~L}(\mathrm{OAC})_{4}\right]^{+}$complexes, assuming $\mathrm{e}_{\sigma / \pi}$ proportional to $1 / \mathrm{R}^{8}$, then the values given Table S4 are obtained. This set of values are then used for calculating the ligand field in both the $\left[\mathrm{Nd}_{2} \mathrm{~L}(\mathrm{OAc})_{4}\right]^{+}$and $\left[\mathrm{Dy}{ }_{2} \mathrm{~L}(\mathrm{OAc})_{4}\right]^{+}$complexes. 
Table S2. Experimental energies and peak heights of the $\left[\mathrm{Nd}_{2} \mathrm{~L}(\mathrm{OAC})_{4}\right]^{+} \mathrm{MCD}$ spectra, together with the calculated energies and the principal components of the wavefunctions.

\begin{tabular}{|c|c|c|c|c|c|}
\hline & \multicolumn{2}{|c|}{ Experiment } & \multicolumn{3}{|r|}{ Calculated } \\
\hline & $\begin{array}{l}\text { Energy } \\
{\left[\mathrm{cm}^{-1}\right]}\end{array}$ & Intensity $^{\mathrm{a}}$ & $\begin{array}{l}\mathrm{Nd} 1 \\
{\left[\mathrm{~cm}^{-1}\right]}\end{array}$ & $\begin{array}{l}\mathrm{Nd} 2 \\
{\left[\mathrm{~cm}^{-1}\right]}\end{array}$ & Wavefunction [\%] (for Nd1) \\
\hline${ }^{4} I_{9 / 2}$ & & & $\begin{array}{r}0 \\
78.0 \\
150.1 \\
391.9 \\
526.5\end{array}$ & $\begin{array}{r}0.0 \\
58.5 \\
103.7 \\
313.8 \\
484.2 \\
\end{array}$ & $\begin{array}{l}95 * 4 \mid(9 / 2) \\
95 * 4 \mid(9 / 2) \\
95 * 4 \mid(9 / 2) \\
95 * 4 \mid(9 / 2) \\
93 * 4 \mid(9 / 2)\end{array}$ \\
\hline${ }^{4} I_{11 / 2}$ & & & $\begin{array}{l}1995.1 \\
2047.0 \\
2138.6 \\
2159.0 \\
2218.3 \\
2288.1 \\
\end{array}$ & $\begin{array}{l}1981.8 \\
2019.8 \\
2048.6 \\
2118.4 \\
2191.8 \\
2224.5 \\
\end{array}$ & $\begin{array}{l}96 * 4 \mid(11 / 2) \\
96 * 4 \mid(11 / 2) \\
96 * 4 \mid(11 / 2) \\
95 * 4 \mid(11 / 2) \\
94 * 4 \mid(11 / 2) \\
95 * 4 \mid(11 / 2) \\
\end{array}$ \\
\hline${ }^{4} I_{13 / 2}$ & & & $\begin{array}{l}3941.4 \\
4001.4 \\
4077.5 \\
4123.9 \\
4165.4 \\
4279.6 \\
4338.3 \\
\end{array}$ & $\begin{array}{l}3928.5 \\
3976.1 \\
3996.4 \\
4077.3 \\
4155.7 \\
4217.5 \\
4264.5 \\
\end{array}$ & $\begin{array}{l}97 * 4 \mid(13 / 2) \\
96 * 4 \mid(13 / 2) \\
96 * 4 I(13 / 2) \\
97 * 4 \mid(13 / 2) \\
97 * 4 I(13 / 2) \\
95 * 4 I(13 / 2) \\
96 * 4 I(13 / 2) \\
\end{array}$ \\
\hline${ }^{4} I_{15 / 2}$ & & & $\begin{array}{l}5805.5 \\
5924.1 \\
6016.0 \\
6133.0 \\
6238.1 \\
6369.5 \\
6540.3 \\
6632.0 \\
\end{array}$ & $\begin{array}{l}5803.2 \\
5916.9 \\
5953.1 \\
6065.7 \\
6223.1 \\
6330.4 \\
6435.3 \\
6543.7 \\
\end{array}$ & $\begin{array}{l}97 * 4 \mid(15 / 2) \\
97 * 4 \mid(15 / 2) \\
97 * 4 \mid(15 / 2) \\
97 * 4 \mid(15 / 2) \\
97 * 4 \mid(15 / 2) \\
97 * 4 \mid(15 / 2) \\
96 * 4 \mid(15 / 2) \\
96 * 4 \mid(15 / 2) \\
\end{array}$ \\
\hline${ }^{4} F_{3 / 2}$ & & & $\begin{array}{l}11516.3 \\
11589.0\end{array}$ & $\begin{array}{l}11485.7 \\
11532.2\end{array}$ & $\begin{array}{l}92 * 4 \mathrm{~F}(3 / 2) \\
93 * 4 \mathrm{~F}(3 / 2)\end{array}$ \\
\hline${ }^{4} \mathrm{~F}_{5 / 2}$ & & & $\begin{array}{l}12502.2 \\
12557.2 \\
12591.3 \\
12628.4 \\
12646.2 \\
12762.1 \\
12829.7 \\
12857.6 \\
\end{array}$ & $\begin{array}{l}12479.0 \\
12515.2 \\
12550.1 \\
12591.2 \\
12605.6 \\
12725.1 \\
12764.1 \\
12812.8 \\
\end{array}$ & $\begin{array}{l}51 * 4 F(5 / 2)+21 * 2 \mathrm{H} 2(9 / 2)+8^{*} 4 \mathrm{~F}(9 / 2)+5 * 2 \mathrm{G} 1(9 / 2) \\
88^{*} 4 \mathrm{~F}(5 / 2) \\
17 * 4 \mathrm{~F}(5 / 2)+10^{*} 4 \mathrm{~F}(9 / 2)+9 * 2 \mathrm{G} 1(9 / 2)+6^{*} 2 \mathrm{G} 2(9 / 2) \\
37 * 4 \mathrm{~F}(5 / 2)+7 * 2 \mathrm{G} 1(9 / 2)+33^{*} 2 \mathrm{H} 2(9 / 2)+6 * 4 \mathrm{~F}(9 / 2) \\
84 * 4 \mathrm{~F}(5 / 2)+6 * 2 \mathrm{H} 2(9 / 2) \\
12 * 4 \mathrm{~F}(9 / 2)+10^{*} 2 \mathrm{G} 1(9 / 2)+7 * 2 \mathrm{G} 2(9 / 2)+7 * 2 \mathrm{H} 1(9 / 2) \\
12 * 4 \mathrm{~F}(9 / 2)+10^{*} 2 \mathrm{G} 1(9 / 2)+7{ }^{*} 2 \mathrm{G} 2(9 / 2)+7 * \mathrm{H} 1(9 / 2) \\
13 * 4 \mathrm{~F}(9 / 2)+10^{*} 2 \mathrm{G} 1(9 / 2)+7 * 2 \mathrm{G} 2(9 / 2)+7 * 2 \mathrm{H} 1(9 / 2)\end{array}$ \\
\hline $\begin{array}{l}{ }^{4} \mathrm{~F}_{9 / 2}+ \\
{ }^{4} \mathrm{~S}_{3 / 2}\end{array}$ & $\begin{array}{l}13661 \\
13689 \\
13717 \\
\end{array}$ & $\begin{array}{l}0.086 \\
0.077 \\
0.046 \\
\end{array}$ & $\begin{array}{l}13427.9 \\
13571.6 \\
13634.0 \\
13646.2 \\
13658.3 \\
13660.8 \\
\end{array}$ & $\begin{array}{l}13402.2 \\
13506.8 \\
13586.0 \\
13600.4 \\
13608.6 \\
13615.6 \\
\end{array}$ & $\begin{array}{l}88 * 4 F(7 / 2) \\
91 * 4 F(7 / 2) \\
82 * 4 F(7 / 2)+8 * 4 S(3 / 2) \\
64 * 4 F(7 / 2)+27 * 4 S(3 / 2) \\
90 * 4 S(3 / 2) \\
60 * 4 S(3 / 2)+32 * 4 F(7 / 2) \\
\end{array}$ \\
\hline${ }^{4} \mathrm{~F}_{9 / 2}$ & $\begin{array}{l}14674 \\
14695 \\
14720 \\
14755 \\
14779 \\
14809 \\
14826 \\
14848\end{array}$ & $\begin{array}{r}0.0025 \\
-0.0023 \\
0.0079 \\
-0.0025 \\
0.0064 \\
-0.0029 \\
0.0018 \\
-0.0025\end{array}$ & $\begin{array}{l}14759.3 \\
14810.4 \\
14851.9 \\
14893.4 \\
14920.9\end{array}$ & $\begin{array}{l}14745.8 \\
14758.2 \\
14794.0 \\
14840.7 \\
14870.4\end{array}$ & $\begin{array}{l}73 * 4 \mathrm{~F}(9 / 2)+19 * 2 \mathrm{H} 2(9 / 2) \\
74 * 4 \mathrm{~F}(9 / 2)+18 * 2 \mathrm{H} 2(9 / 2) \\
74 * 4 \mathrm{~F}(9 / 2)+19 * 2 \mathrm{H} 2(9 / 2) \\
73 * 4 \mathrm{~F}(9 / 2)+19 * 2 \mathrm{H} 2(9 / 2) \\
74 * 4 \mathrm{~F}(9 / 2)+19 * 2 \mathrm{H} 2(9 / 2)\end{array}$ \\
\hline
\end{tabular}




\begin{tabular}{|c|c|c|c|c|c|}
\hline & $\begin{array}{l}14864 \\
14875 \\
14903 \\
14931 \\
\end{array}$ & $\begin{array}{r}0.0022 \\
0.0037 \\
-0.0011 \\
0.00095 \\
\end{array}$ & & & \\
\hline \multirow{6}{*}{${ }^{2} \mathrm{H}_{11 / 2}$} & 15936 & 0.0027 & 15991.9 & 15956.2 & $12 * 2 \mathrm{H} 1(11 / 2)+78 * 2 \mathrm{H} 2(11 / 2)+6 * 4 \mathrm{G}(11 / 2)$ \\
\hline & 15981 & 0.003 & 16002.5 & 15970.3 & $12 * 2 \mathrm{H} 1(11 / 2)+78 * 2 \mathrm{H} 2(11 / 2)+6 * 4 \mathrm{G}(11 / 2)$ \\
\hline & 16013 & 0.0093 & 16020.6 & 15979.4 & $12 * 2 \mathrm{H} 1(11 / 2)+78 * 2 \mathrm{H} 2(11 / 2)+5 * 4 \mathrm{G}(11 / 2)$ \\
\hline & & & 16028.6 & 15998.1 & $12 * 2 \mathrm{H} 1(11 / 2)+78 * 2 \mathrm{H} 2(11 / 2)+5 * 4 \mathrm{G}(11 / 2)$ \\
\hline & & & 16070.5 & 16026.6 & $12 * 2 \mathrm{H} 1(11 / 2)+78 * 2 \mathrm{H} 2(11 / 2)+5 * 4 \mathrm{G}(11 / 2)$ \\
\hline & & & 16117.7 & 16069.9 & $12 * 2 \mathrm{H} 1(11 / 2)+79 * 2 \mathrm{H} 2(11 / 2)+5 * 4 \mathrm{G}(11 / 2)$ \\
\hline \multirow{8}{*}{$\begin{array}{l}{ }^{4} G_{5 / 2}+ \\
{ }^{2} G_{7 / 2}\end{array}$} & 17065 & -0.075 & 17074.0 & 17073.7 & $78 * 4 G(5 / 2)+10 * 4 G(7 / 2)$ \\
\hline & 17116 & -0.52 & 17130.3 & 17120.9 & $61 * 4 G(5 / 2)+18 * 4 G(7 / 2)+9 * 2 G 1(7 / 2)+6 * 2 G 2(7 / 2)$ \\
\hline & 17197 & -0.23 & 17332.9 & 17227.0 & $71 * 4 \mathrm{G}(5 / 2)+11 * 4 \mathrm{G}(7 / 2)+7 * 2 \mathrm{G} 1(7 / 2)+5 * 2 \mathrm{G} 2(7 / 2)$ \\
\hline & 17249 & -0.19 & 17395.3 & 17343.3 & $34 * 4 G(7 / 2)+27 * 2 G 1(7 / 2)+20 * 2 G 2(7 / 2)+10 * 4 G(5 / 2)$ \\
\hline & 17316 & -0.12 & 17417.4 & 17375.6 & $35 * 4 G(7 / 2)+28 * 2 G 1(7 / 2)+21 * 2 G 2(7 / 2)+7 * 4 G(5 / 2)$ \\
\hline & 17369 & -0.075 & 17441.3 & 17385.2 & $28 * 4 G(7 / 2)+27 * 2 G 1(7 / 2)+20 * 2 G 2(7 / 2)+17 * 4 G(5 / 2)$ \\
\hline & 17422 & -0.079 & 17507.7 & 17456.9 & $44 * 4 G(5 / 2)+18 * 2 G 1(7 / 2)+17 * 4 G(7 / 2)+13 * 2 G 2(7 / 2)$ \\
\hline & 17483 & -0.054 & & & \\
\hline \multirow{5}{*}{${ }^{4} G_{7 / 2}$} & 18940 & -0.034 & 18988.5 & 18972.1 & $52 * 4 G(7 / 2)+21 * 2 G 1(7 / 2)+13 * 2 G 2(7 / 2)+5 * 2 K(13 / 2)$ \\
\hline & 19003 & -0.095 & 19057.4 & 19037.5 & $51 * 4 G(7 / 2)+20 * 2 G 1(7 / 2)+12 * 2 G 2(7 / 2)+6 * 2 K(13 / 2)$ \\
\hline & 19084 & -0.07 & 19125.9 & 19116.3 & $51 * 4 G(7 / 2)+17 * 2 G 1(7 / 2)+10 * 2 G 2(7 / 2)+9 * 4 G(9 / 2)$ \\
\hline & 19121 & 0.016 & 19230.2 & 19151.1 & $55 * 4 G(7 / 2)+21 * 2 G 1(7 / 2)+12 * 2 G 2(7 / 2)+5 * 2 K(13 / 2)$ \\
\hline & 19194 & -0.022 & & & \\
\hline \multirow{12}{*}{$\begin{array}{l}{ }^{4} G_{9 / 2}+ \\
{ }^{2} K_{13 / 2}\end{array}$} & 19484 & 0.012 & 19454.9 & 19438.6 & $67 * 2 K(13 / 2)+19 * 4 G(9 / 2)$ \\
\hline & 19532 & -0.0097 & 19519.8 & 19470.1 & $48 * 4 G(9 / 2)+31 * 2 K(13 / 2)+5 * 2 G 1(9 / 2)$ \\
\hline & 19589 & 0.032 & 19532.9 & 19478.3 & $58 * 4 G(9 / 2)+17 * 2 K(13 / 2)+7 * 2 G 1(9 / 2)+6 * 2 G 2(9 / 2)$ \\
\hline & 19620 & 0.032 & 19557.7 & 19536.1 & $53 * 4 G(9 / 2)+25 * 2 K(13 / 2)+5 * 2 G 2(9 / 2)+5 * 2 G 1(9 / 2)$ \\
\hline & 19734 & 0.03 & 19580.6 & 19548.0 & $52 * 4 G(9 / 2)+26 * 2 K(13 / 2)$ \\
\hline & 19783 & 0.029 & 19607.6 & 19557.8 & $48 * 4 G(9 / 2)+31 * 2 K(13 / 2)$ \\
\hline & 19861 & 0.026 & 19671.7 & 19591.6 & $73 * 2 K(13 / 2)+16 * 4 G(9 / 2)$ \\
\hline & 19980 & 0.015 & 19681.1 & 19654.8 & $22 * 4 G(9 / 2)+56 * 2 K(13 / 2)+5 * 4 G(7 / 2)$ \\
\hline & & & 19756.6 & 19687.2 & $11 * 4 G(9 / 2)+69 * 2 K(13 / 2)+6 * 4 G(7 / 2)$ \\
\hline & & & 19848.2 & 19783.0 & $83 * 2 K(13 / 2)+7 * 4 G(9 / 2)$ \\
\hline & & & 19888.7 & 19823.2 & $86 * 2 K(13 / 2)+7 * 4 G(9 / 2)$ \\
\hline & & & 19943.3 & 19874.2 & $90 * 2 \mathrm{~K}(13 / 2)+5 * 4 \mathrm{G}(9 / 2)$ \\
\hline \multirow{7}{*}{$\begin{array}{l}{ }^{2} G_{9 / 2}+ \\
{ }^{2} P_{3 / 2}\end{array}$} & 21020 & -0.01 & 21021.5 & 21003.4 & $15 * 4 G(9 / 2)+8 * 4 G(11 / 2)+31 * 2 G 1(9 / 2)+6 * 4 F(9 / 2)$ \\
\hline & 21075 & 0.0081 & 21046.7 & 21025.1 & $15 * 4 G(9 / 2)+8 * 4 G(11 / 2)+29 * 2 G 1(9 / 2)+5 * 4 F(9 / 2)$ \\
\hline & & & 21055.6 & 21062.9 & $16 * 4 G(9 / 2)+11 * 4 G(11 / 2)+26 * 2 G 1(9 / 2)+5 * 4 F(9 / 2)$ \\
\hline & & & 21087.4 & 21080.8 & $15 * 4 G(9 / 2)+8 * 2 D 1(3 / 2)+5 * 2 P(3 / 2)+5 * 4 F(9 / 2)$ \\
\hline & 21198 & -0.011 & 21202.2 & 21128.4 & $21 * 4 \mathrm{G}(9 / 2)+31 * 2 \mathrm{G} 1(9 / 2)+19 * 2 \mathrm{G} 2(9 / 2)+6 * 4 \mathrm{~F}(9 / 2)$ \\
\hline & & & 21245.8 & 21201.4 & $27 * 2 \mathrm{D} 1(3 / 2)+25 * 2 \mathrm{P}(3 / 2)+18 * 2 \mathrm{~K}(15 / 2)+7 * 2 \mathrm{G} 1(9 / 2)$ \\
\hline & & & 21258.1 & 21215.8 & $26 * 2 \mathrm{D} 1(3 / 2)+23 * 2 \mathrm{P}(3 / 2)+10 * 2 \mathrm{G} 1(9 / 2)+6 * 2 \mathrm{G} 2(9 / 2)$ \\
\hline \multirow{15}{*}{$\begin{array}{l}{ }^{2} \mathrm{~K}_{15 / 2}+ \\
{ }^{4} \mathrm{G}_{11 / 2}\end{array}$} & 21345 & -0.0022 & 21380.6 & 21341.0 & $68 * 4 G(11 / 2)+15 * 2 K(15 / 2)$ \\
\hline & & & 21439.6 & 21430.7 & $68 * 4 G(11 / 2)+15 * 2 K(15 / 2)$ \\
\hline & & & 21537.6 & 21485.9 & $64 * 2 \mathrm{~K}(15 / 2)+15 * 4 \mathrm{G}(11 / 2)$ \\
\hline & & & 21581.5 & 21536.0 & $72 * 4 G(11 / 2)+17 * 2 K(15 / 2)$ \\
\hline & & & 21620.2 & 21563.1 & $73 * 4 G(11 / 2)+16 * 2 K(15 / 2)$ \\
\hline & 21657 & -0.0094 & 21648.8 & 21604.0 & $62 * 2 K(15 / 2)+19 * 4 G(11 / 2)$ \\
\hline & & & 21712.9 & 21655.0 & $50 * 4 G(11 / 2)+34 * 2 K(15 / 2)$ \\
\hline & 21882 & 0.0048 & 21750.7 & 21674.2 & $48 * 2 K(15 / 2)+35 * 4 G(11 / 2)$ \\
\hline & & & 21772.8 & 21712.2 & $44 * 2 K(15 / 2)+38 * 4 G(11 / 2)$ \\
\hline & & & 21802.0 & 21734.0 & $69 * 2 K(15 / 2)+12 * 4 G(11 / 2)$ \\
\hline & & & 21832.6 & 21760.4 & $71 * 2 K(15 / 2)+12 * 4 G(11 / 2)$ \\
\hline & & & 21868.0 & 21799.9 & $75 * 2 K(15 / 2)+10 * 4 G(11 / 2)$ \\
\hline & & & 21907.1 & 21841.0 & $68 * 2 K(15 / 2)+12 * 4 G(11 / 2)$ \\
\hline & & & 21964.2 & 21873.7 & $68 * 2 K(15 / 2)+17 * 4 G(11 / 2)$ \\
\hline & 23283 & -0.0069 & 23330.8 & 23285.5 & $91 * 2 \mathrm{P}(1 / 2)+7 * 4 \mathrm{D}(1 / 2)$ \\
\hline
\end{tabular}




\begin{tabular}{|c|c|c|c|c|c|}
\hline${ }^{2} \mathrm{P}_{1 / 2}$ & 23406 & -0.0093 & & & \\
\hline \multirow{3}{*}{${ }^{2} D_{5 / 2}$} & 24390 & -0.0021 & 23849.8 & 23816.2 & $96 * 2 \mathrm{D} 1(5 / 2)$ \\
\hline & 24691 & 0.0010 & 23911.7 & 23871.3 & $95 * 2 \mathrm{D} 1(5 / 2)$ \\
\hline & 24876 & 0.0023 & 23985.8 & 23910.4 & $96 * 2 \mathrm{D} 1(5 / 2)$ \\
\hline \multirow{2}{*}{$\begin{array}{l}{ }^{2} \mathrm{D}_{3 / 2}+ \\
{ }^{2} \mathrm{P}_{3 / 2}\end{array}$} & & & 26232.5 & 26193.0 & $51 * 2 \mathrm{P}(3 / 2)+40 * 2 \mathrm{D} 1(3 / 2)$ \\
\hline & & & 26273.8 & 26229.3 & $51 * 2 \mathrm{P}(3 / 2)+39 * 2 \mathrm{D} 1(3 / 2)$ \\
\hline \multirow{6}{*}{$\begin{array}{l}{ }^{4} D_{5 / 2}+ \\
{ }^{4} D_{3 / 2}\end{array}$} & 27777 & 0.016 & 27996.5 & 28005.7 & $47 * 4 D(3 / 2)+33 * 4 D(5 / 2)+7 * 2 D 2(3 / 2)+7 * 2 D 2(5 / 2)$ \\
\hline & 27924 & 0.038 & 28090.4 & 28046.7 & $74 * 4 D(3 / 2)+12 * 2 D 2(3 / 2)+6 * 4 D(5 / 2)$ \\
\hline & 28026 & 0.064 & 28220.9 & 28143.9 & $51 * 4 D(5 / 2)+27 * 4 D(3 / 2)+11 * 2 D 2(5 / 2)$ \\
\hline & 28152 & 0.037 & 28296.4 & 28240.4 & $70 * 4 D(5 / 2)+15 * 2 D 2(5 / 2)+7 * 4 D(3 / 2)$ \\
\hline & 28288 & 0.022 & 28411.0 & 28361.3 & $73 * 4 D(5 / 2)+17 * 2 D 2(5 / 2)$ \\
\hline & 28384 & 0.032 & & & \\
\hline \multirow[t]{2}{*}{${ }^{4} D_{1 / 2}$} & 28620 & -0.028 & 28662.2 & 28603.5 & $86 * 4 D(1 / 2)+6 * 2 P(1 / 2)$ \\
\hline & 28810 & -0.031 & & & \\
\hline \multirow{6}{*}{${ }^{2} I_{11 / 2}$} & 28943 & 0.0017 & 29209.3 & 29196.0 & $78 * 2 \mathrm{I}(11 / 2)+14 * 2 \mathrm{H} 1(11 / 2)$ \\
\hline & 29061 & -0.0026 & 29273.0 & 29236.8 & $79 * 2 \mathrm{I}(11 / 2)+14 * 2 \mathrm{H} 1(11 / 2)$ \\
\hline & 29154 & 0.0014 & 29336.1 & 29329.4 & $79 * 2 I(11 / 2)+14 * 2 \mathrm{H} 1(11 / 2)$ \\
\hline & & & 29424.3 & 29406.7 & $78 * 2 I(11 / 2)+14 * 2 \mathrm{H} 1(11 / 2)$ \\
\hline & & & 29493.8 & 29496.4 & $77 * 2 \mid(11 / 2)+14 * 2 \mathrm{H} 1(11 / 2)$ \\
\hline & & & 29692.6 & 29531.8 & $77 * 2 \mathrm{l}(11 / 2)+14 * 2 \mathrm{H} 1(11 / 2)$ \\
\hline
\end{tabular}


Table S3. Experimental energies and peak heights of the $\left[D y_{2} L(O A c)_{4}\right]^{+} M C D$ spectra, together with the calculated energies and the principal components of the wavefunctions.

\begin{tabular}{|c|c|c|c|c|c|}
\hline & \multicolumn{2}{|c|}{ Experiment } & \multicolumn{3}{|r|}{ Calculated } \\
\hline & $\begin{array}{l}\text { Energy } \\
{\left[\mathrm{cm}^{-1}\right]}\end{array}$ & Intensity & $\begin{array}{c}\text { Dy1 } \\
{\left[\mathrm{cm}^{-1}\right]}\end{array}$ & $\begin{array}{c}\text { Dy2 } \\
{\left[\mathrm{cm}^{-1}\right]}\end{array}$ & Wavefunction [\%] (for Dy1) \\
\hline \multirow{8}{*}{${ }^{6} \mathrm{H}_{15 / 2}$} & & & 0.0 & 0.0 & $93 * 6 \mathrm{H}(15 / 2)$ \\
\hline & & & 38.8 & 43.7 & $93 * 6 \mathrm{H}(15 / 2)$ \\
\hline & & & 105.1 & 81.4 & $93 * 6 \mathrm{H}(15 / 2)$ \\
\hline & & & 148.9 & 135.2 & $93 * 6 \mathrm{H}(15 / 2)$ \\
\hline & & & 265.2 & 213.8 & $93^{*} 6 \mathrm{H}(15 / 2)$ \\
\hline & & & 325.2 & 309.5 & $93 * 6 \mathrm{H}(15 / 2)$ \\
\hline & & & 416.8 & 362.3 & $93 * 6 \mathrm{H}(15 / 2)$ \\
\hline & & & 498.2 & 431.9 & $93 * 6 \mathrm{H}(15 / 2)$ \\
\hline \multirow{7}{*}{${ }^{6} \mathrm{H}_{15 / 2}$} & & & 3551.5 & 3562.8 & $95 * 6 \mathrm{H}(13 / 2)$ \\
\hline & & & 3607.8 & 3581.9 & $95 * 6 \mathrm{H}(13 / 2)$ \\
\hline & & & 3639.8 & 3617.3 & $95 * 6 \mathrm{H}(13 / 2)$ \\
\hline & & & 3719.0 & 3689.1 & $95 * 6 \mathrm{H}(13 / 2)$ \\
\hline & & & 3754.5 & 3718.8 & $95 * 6 \mathrm{H}(13 / 2)$ \\
\hline & & & 3795.2 & 3748.9 & $95 * 6 \mathrm{H}(13 / 2)$ \\
\hline & & & 3820.9 & 3771.5 & $95 * 6 \mathrm{H}(13 / 2)$ \\
\hline \multirow{6}{*}{${ }^{6} \mathrm{H}_{11 / 2}$} & & & 5909.6 & 5890.0 & $93 * 6 \mathrm{H}(11 / 2)$ \\
\hline & & & 5948.8 & 5928.7 & $90 * 6 \mathrm{H}(11 / 2)$ \\
\hline & & & 5974.2 & 5959.0 & $91 * 6 \mathrm{H}(11 / 2)$ \\
\hline & & & 6000.3 & 5979.8 & $85 * 6 H(11 / 2)+7 * 6 F(11 / 2)$ \\
\hline & & & 6035.7 & 6038.0 & $87^{*} 6 \mathrm{H}(11 / 2)+5 * 6 \mathrm{~F}(11 / 2)$ \\
\hline & & & 6143.8 & 6059.9 & $89 * 6 \mathrm{H}(11 / 2)$ \\
\hline \multirow{11}{*}{$\begin{array}{l}{ }^{6} \mathrm{H}_{9 / 2}+ \\
{ }^{6} \mathrm{~F}_{11 / 2}\end{array}$} & & & 7603.6 & 7591.6 & $57 * 6 \mathrm{H}(9 / 2)+34 * 6 \mathrm{~F}(11 / 2)$ \\
\hline & & & 7641.1 & 7637.1 & $64 * 6 H(9 / 2)+27 * 6 F(11 / 2)$ \\
\hline & & & 7689.1 & 7695.5 & $54 * 6 \mathrm{H}(9 / 2)+36 * 6 \mathrm{~F}(11 / 2)$ \\
\hline & & & 7763.3 & 7751.6 & $45 * 6 \mathrm{~F}(11 / 2)+45 * 6 \mathrm{H}(9 / 2)$ \\
\hline & & & 7819.4 & 7788.5 & $79 * 6 \mathrm{~F}(11 / 2)+14 * 6 \mathrm{H}(9 / 2)$ \\
\hline & & & 7840.7 & 7833.8 & $73 * 6 \mathrm{~F}(11 / 2)+18 * 6 \mathrm{H}(9 / 2)$ \\
\hline & & & 7920.4 & 7864.6 & $51 * 6 F(11 / 2)+39 * 6 H(9 / 2)$ \\
\hline & & & 8012.1 & 7953.7 & $54 * 6 \mathrm{H}(9 / 2)+36 * 6 \mathrm{~F}(11 / 2)$ \\
\hline & & & 8062.0 & 7989.9 & $58 * 6 \mathrm{~F}(11 / 2)+30 * 6 \mathrm{H}(9 / 2)$ \\
\hline & & & 8173.2 & 8103.4 & $45 * 6 F(11 / 2)+42 * 6 H(9 / 2)$ \\
\hline & & & 8207.8 & 8172.2 & $53 * 6 \mathrm{~F}(11 / 2)+33 * 6 \mathrm{H}(9 / 2)+6 * 6 \mathrm{H}(11 / 2)$ \\
\hline \multirow{9}{*}{$\begin{array}{l}{ }^{6} \mathrm{H}_{7 / 2}+ \\
{ }^{6} \mathrm{~F}_{9 / 2}\end{array}$} & & & 8937.3 & 8928.3 & $56 * 6 \mathrm{~F}(9 / 2)+31 * 6 \mathrm{H}(7 / 2)$ \\
\hline & 8980.7 & 0.051 & 9043.8 & 9029.3 & $45 * 6 F(9 / 2)+43 * 6 H(7 / 2)$ \\
\hline & 9033.4 & 0.14 & 9103.8 & 9075.9 & $44 * 6 F(9 / 2)+43 * 6 H(7 / 2)$ \\
\hline & 9074.4 & 0.22 & 9168.1 & 9165.8 & $70 * 6 F(9 / 2)+14 * 6 H(7 / 2)+5 * 4 F 3(9 / 2)$ \\
\hline & 9132.4 & 0.19 & 9193.9 & 9182.4 & $59 * 6 F(9 / 2)+26 * 6 H(7 / 2)$ \\
\hline & 9250.7 & 0.096 & 9309.5 & 9231.5 & $55 * 6 F(9 / 2)+32 * 6 H(7 / 2)$ \\
\hline & 9416.2 & 0.036 & 9347.2 & 9308.9 & $43 * 6 \mathrm{~F}(9 / 2)+42 * 6 \mathrm{H}(7 / 2)$ \\
\hline & & & 9515.5 & 9448.7 & $55 * 6 \mathrm{H}(7 / 2)+30 * 6 \mathrm{~F}(9 / 2)$ \\
\hline & & & 9593.3 & 9543.6 & $66 * 6 \mathrm{H}(7 / 2)+21 * 6 \mathrm{~F}(9 / 2)$ \\
\hline \multirow{3}{*}{${ }^{6} \mathrm{H}_{5 / 2}$} & & & 10218.6 & 10230.8 & $85 * 6 \mathrm{H}(5 / 2)$ \\
\hline & & & 10336.6 & 10293.7 & $77 * 6 \mathrm{H}(5 / 2)+11 * 6 \mathrm{~F}(7 / 2)$ \\
\hline & & & 10496.6 & 10422.2 & $81 * 6 \mathrm{H}(5 / 2)$ \\
\hline \multirow{6}{*}{${ }^{6} \mathrm{~F}_{7 / 2}$} & 10962 & -0.0059 & 11091.0 & 11048.8 & $89 * 6 F(7 / 2)$ \\
\hline & 10995 & 0.15 & 11121.8 & 11108.4 & $88 * 6 F(7 / 2)$ \\
\hline & 11041 & 0.32 & 11224.1 & 11186.6 & $87^{*} 6 \mathrm{~F}(7 / 2)$ \\
\hline & 11114 & -0.01 & 11306.3 & 11221.9 & $79 * 6 F(7 / 2)+10 * 6 H(5 / 2)$ \\
\hline & 11152 & 0.049 & & & \\
\hline & 12400 & 0.55 & 12509.4 & 12478.5 & $91 * 6 F(5 / 2)$ \\
\hline
\end{tabular}




\begin{tabular}{|c|c|c|c|c|c|}
\hline${ }^{6} \mathrm{~F}_{5 / 2}$ & 12423 & 0.55 & $\begin{array}{l}12560.8 \\
12598.5\end{array}$ & $\begin{array}{l}12516.2 \\
12555.8 \\
\end{array}$ & $\begin{array}{l}91 * 6 \mathrm{~F}(5 / 2) \\
90 * 6 \mathrm{~F}(5 / 2)\end{array}$ \\
\hline${ }^{6} F_{3 / 2}$ & $\begin{array}{l}13202 \\
13237\end{array}$ & $\begin{array}{l}0.34 \\
0.25\end{array}$ & $\begin{array}{l}13340.4 \\
13348.7\end{array}$ & $\begin{array}{l}13298.2 \\
13310.2\end{array}$ & $\begin{array}{l}89 * 6 F(3 / 2) \\
89 * 6 F(3 / 2)\end{array}$ \\
\hline${ }^{6} \mathrm{~F}_{1 / 2}$ & & & 13890.1 & 13851.8 & $90 * 6 \mathrm{~F}(1 / 2)$ \\
\hline \multirow{5}{*}{${ }^{4} F_{9 / 2}$} & 20976 & 0.038 & 20996.3 & 21007.9 & $9 * 4 F 1(9 / 2)+43 * 4 F 3(9 / 2)+8 * 6 F(9 / 2)+8 * 4 F 4(9 / 2)$ \\
\hline & 21016 & 0.051 & 21065.7 & 21039.5 & $9 * 4 F 1(9 / 2)+43 * 4 F 3(9 / 2)+8 * 6 F(9 / 2)+8 * 4 F 4(9 / 2)$ \\
\hline & 21104 & 0.11 & 21118.0 & 21108.9 & $9 * 4 F 1(9 / 2)+43 * 4 F 3(9 / 2)+8 * 6 F(9 / 2)+8 * 4 F 4(9 / 2)$ \\
\hline & 21198 & 0.092 & 21266.3 & 21216.5 & $9 * 4 F 1(9 / 2)+43 * 4 F 3(9 / 2)+8 * 6 F(9 / 2)+8 * 4 F 4(9 / 2)$ \\
\hline & 21275 & 0.052 & 21422.9 & 21408.7 & $9 * 4 F 1(9 / 2)+41 * 4 F 3(9 / 2)+8 * 4 F 4(9 / 2)+7 * 6 F(9 / 2)$ \\
\hline \multirow{8}{*}{$\begin{array}{l}{ }^{4} I_{15 / 2}+ \\
{ }^{4} K_{15 / 2}\end{array}$} & & & 21891.3 & 21924.2 & $42 * 4 I 3(15 / 2)+11 * 4 K 1(15 / 2)+9 * 4 \mid 1(15 / 2)$ \\
\hline & & & 21981.0 & 21984.5 & $41 * 4 I 3(15 / 2)+11 * 4 K 1(15 / 2)+8 * 4 I 1(15 / 2)$ \\
\hline & 21982 & -0.0077 & 22077.0 & 22031.4 & $38 * 4 I 3(15 / 2)+10 * 4 K 1(15 / 2)+8 * 4 I 1(15 / 2)$ \\
\hline & 22094 & -0.012 & 22167.9 & 22133.1 & $43 * 4 I 3(15 / 2)+11 * 4 K 1(15 / 2)+9 * 4 I 1(15 / 2)$ \\
\hline & 22206 & 0.023 & 22216.0 & 22212.5 & $43 * 4 I 3(15 / 2)+11 * 4 K 1(15 / 2)+9 * 4 I 1(15 / 2)$ \\
\hline & 22326 & 0.035 & 22289.3 & 22281.8 & $42 * 4 I 3(15 / 2)+12 * 4 K 1(15 / 2)+9 * 4 I 1(15 / 2)$ \\
\hline & & & 22389.2 & 22326.1 & $43 * 4 I 3(15 / 2)+11 * 4 K 1(15 / 2)+9 * 4 I 1(15 / 2)$ \\
\hline & & & 22447.4 & 22391.7 & $44 * 4 I 3(15 / 2)+11 * 4 K 1(15 / 2)+9 * 4 I 1(15 / 2)$ \\
\hline \multirow{6}{*}{${ }^{4} \mathrm{G}_{11 / 2}$} & & & 23379.5 & 23368.0 & $43 * 4 \mathrm{G} 4(11 / 2)+24 * 4 \mathrm{G} 1(11 / 2)+10 * 4 \mathrm{G} 2(11 / 2)$ \\
\hline & & & 23456.6 & 23442.2 & $41 * 4 \mathrm{G} 4(11 / 2)+23 * 4 \mathrm{G} 1(11 / 2)+10 * 4 \mathrm{G} 2(11 / 2)$ \\
\hline & 23397 & -0.016 & 23487.3 & 23466.6 & $42 * 4 \mathrm{G} 4(11 / 2)+23 * 4 \mathrm{G} 1(11 / 2)+11 * 4 \mathrm{G} 2(11 / 2)$ \\
\hline & 23513 & -0.0081 & 23509.2 & 23491.6 & $42 * 4 \mathrm{G} 4(11 / 2)+23 * 4 \mathrm{G} 1(11 / 2)+11 * 4 \mathrm{G} 2(11 / 2)$ \\
\hline & & & 23536.0 & 23529.7 & $42 * 4 \mathrm{G} 4(11 / 2)+23 * 4 \mathrm{G} 1(11 / 2)+11 * 4 \mathrm{G} 2(11 / 2)$ \\
\hline & & & 23652.6 & 23575.1 & $43 * 4 \mathrm{G} 4(11 / 2)+24 * 4 \mathrm{G} 1(11 / 2)+11 * 4 \mathrm{G} 2(11 / 2)$ \\
\hline \multirow{11}{*}{${ }^{4} M_{21 / 2}$} & & & 24893.6 & 24894.4 & $89 * 4 M(21 / 2)+5 * 2 N(21 / 2)$ \\
\hline & & & 24933.6 & 24899.8 & $89 * 4 M(21 / 2)+5 * 2 N(21 / 2)$ \\
\hline & 25012 & 0.039 & 24952.2 & 24947.1 & $88 * 4 M(21 / 2)+5 * 2 N(21 / 2)$ \\
\hline & & & 25004.1 & 24995.0 & $87 * 4 M(21 / 2)+5 * 2 N(21 / 2)$ \\
\hline & & & 25034.7 & 25071.6 & $88 * 4 M(21 / 2)+6 * 2 N(21 / 2)$ \\
\hline & & & 25122.4 & 25151.1 & $84 * 4 M(21 / 2)+5 * 2 N(21 / 2)$ \\
\hline & & & 25227.0 & 25196.7 & $81 * 4 M(21 / 2)+5 * 2 N(21 / 2)$ \\
\hline & & & 25289.4 & 25237.8 & $79 * 4 M(21 / 2)+5 * 2 N(21 / 2)$ \\
\hline & & & 25330.6 & 25264.3 & $75 * 4 M(21 / 2)+5 * 413(13 / 2)+5 * 2 N(21 / 2)$ \\
\hline & & & 25338.5 & 25285.9 & $81 * 4 M(21 / 2)+5 * 2 N(21 / 2)$ \\
\hline & & & 25372.1 & 25339.5 & $81 * 4 M(21 / 2)+5 * 2 N(21 / 2)$ \\
\hline \multirow{20}{*}{$\begin{array}{l}{ }^{4} L_{17 / 2} \\
{ }^{4} K_{17 / 2}+ \\
{ }^{4} I_{13 / 2}+ \\
{ }^{4} K_{13 / 2}+ \\
{ }^{4} F_{7 / 2}\end{array}$} & & & 25572.4 & 25568.8 & $29 * 4 I 3(13 / 2)+12 * 4 F 3(7 / 2)+8 * 4 K 1(13 / 2)+5 * 4 I 1(13 / 2)$ \\
\hline & & & 25592.7 & 25599.8 & $34 * 4 I 3(13 / 2)+8 * 4 F 3(7 / 2)+10 * 4 K 1(13 / 2)+6 * 4 I 1(13 / 2)$ \\
\hline & 25693 & -0.15 & 25639.5 & 25638.5 & $33 * 4 I 3(13 / 2)+7 * 4 F 3(7 / 2)+10 * 4 K 1(13 / 2)+6 * 4 I 1(13 / 2)$ \\
\hline & 25766 & -0.11 & 25709.1 & 25670.6 & $16 * 4 F 3(7 / 2)+16 * 4 I 3(13 / 2)+7 * 4 K 1(17 / 2)+5 * 4 L(17 / 2)$ \\
\hline & 25873 & -0.1 & 25750.6 & 25711.5 & $25 * 4 K 1(17 / 2)+18 * 4 L(17 / 2)+17 * 4 M(17 / 2)+6 * 4 M(21 / 2)$ \\
\hline & 26001 & -0.036 & 25762.1 & 25729.6 & $26 * 4 K 1(17 / 2)+19 * 4 L(17 / 2)+17 * 4 M(17 / 2)+6 * 4 M(21 / 2)$ \\
\hline & 26137 & 0.027 & 25787.0 & 25749.7 & $10 * 4 I 3(13 / 2)+18 * 4 K 1(17 / 2)+13 * 4 L(17 / 2)+9 * 4 F 3(7 / 2)$ \\
\hline & 26219 & 0.026 & 25803.8 & 25767.0 & $10 * 4 I 3(13 / 2)+18 * 4 K 1(17 / 2)+12 * 4 L(17 / 2)+9 * 4 F 3(7 / 2)$ \\
\hline & & & 25817.5 & 25778.0 & $20 * 4 I 3(13 / 2)+14 * 4 K 1(17 / 2)+6 * 4 K 1(13 / 2)+6 * 4 F 3(7 / 2)$ \\
\hline & & & 25842.8 & 25821.5 & $22 * 4 I 3(13 / 2)+11 * 4 \mathrm{~F} 3(7 / 2)+7 * 4 K 1(17 / 2)+6 * 4 K 1(13 / 2)$ \\
\hline & & & 25871.8 & 25845.4 & $25 * 4 \mathrm{~K} 1(17 / 2)+15 * 4 \mathrm{~L}(17 / 2)+13 * 4 \mathrm{M}(17 / 2)+11 * 4 \mathrm{I} 3(13 / 2)$ \\
\hline & & & 25892.1 & 25858.7 & $14 * 4 I 3(13 / 2)+19 * 4 K 1(17 / 2)+11 * 4 L(17 / 2)+6 * 4 F 3(7 / 2)$ \\
\hline & & & 25901.5 & 25882.8 & $9 * 4 I 3(13 / 2)+25 * 4 K 1(17 / 2)+14 * 4 L(17 / 2)+5 * 4 F 3(7 / 2)$ \\
\hline & & & 25919.8 & 25891.4 & $27 * 4 K 1(17 / 2)+15 * 4 L(17 / 2)+11 * 4 M(17 / 2)+7 * 4 I 3(13 / 2)$ \\
\hline & & & 25930.0 & 25895.0 & $21 * 4 K 1(17 / 2)+16 * 4 I 3(13 / 2)+11 * 4 L(17 / 2)+9 * 4 M(17 / 2)$ \\
\hline & & & 25947.9 & 25908.0 & $14 * 4 I 3(13 / 2)+16 * 4 K 1(17 / 2)+10 * 4 L(17 / 2)+8 * 4 F 3(7 / 2)$ \\
\hline & & & 25961.8 & 25921.0 & $14 * 4 I 3(13 / 2)+19 * 4 K 1(17 / 2)+11 * 4 L(17 / 2)+7 * 4 F 3(7 / 2)$ \\
\hline & & & 25987.7 & 25946.4 & $25 * 4 K 1(17 / 2)+15 * 4 L(17 / 2)+11 * 4 M(17 / 2)+10 * 4 I 3(13 / 2)$ \\
\hline & & & 26026.3 & 25978.5 & $30 * 4|3(13 / 2)+9 * 4 K 1(13 / 2)+13 * 4 K 1(17 / 2)+5 * 4| 1(13 / 2)$ \\
\hline & & & 26056.7 & 26006.5 & $31 * 4 I 3(13 / 2)+9 * 4 K 1(13 / 2)+5 * 4 I 1(13 / 2)+5 * 4 K 1(17 / 2)$ \\
\hline \multirow{2}{*}{${ }^{4} \mathrm{M}_{19 / 2}+$} & & & 26100.7 & 26104.2 & $54 * 4 M(19 / 2)+32 * 4 L(19 / 2)$ \\
\hline & & & 26134.4 & 26112.8 & $50 * 4 M(19 / 2)+29 * 4 L(19 / 2)$ \\
\hline
\end{tabular}




\begin{tabular}{|c|c|c|c|c|c|}
\hline${ }^{4} L_{19 / 2}$ & & & $\begin{array}{l}26174.7 \\
26248.3 \\
26326.4 \\
26398.8 \\
26475.9 \\
26606.3 \\
26624.8 \\
26639.8 \\
\end{array}$ & $\begin{array}{l}26148.2 \\
26189.3 \\
26295.9 \\
26366.0 \\
26461.5 \\
26530.6 \\
26548.7 \\
26577.5 \\
\end{array}$ & $\begin{array}{l}27 * 4 \mathrm{~L}(19 / 2)+47 * 4 \mathrm{M}(19 / 2)+8 * 4 I 3(13 / 2) \\
28 * 4 \mathrm{~L}(19 / 2)+47 * 4 \mathrm{M}(19 / 2)+5 * 4 I 3(13 / 2) \\
55 * 4 \mathrm{M}(19 / 2)+33 * 4 \mathrm{~L}(19 / 2) \\
55 * 4 \mathrm{M}(19 / 2)+34 * 4 \mathrm{~L}(19 / 2) \\
55 * 4 \mathrm{M}(19 / 2)+31 * 4 \mathrm{~L}(19 / 2) \\
55 * 4 \mathrm{M}(19 / 2)+35 * 4 \mathrm{~L}(19 / 2) \\
56 * 4 \mathrm{M}(19 / 2)+34 * 4 \mathrm{~L}(19 / 2) \\
55 * 4 \mathrm{M}(19 / 2)+35 * 4 \mathrm{~L}(19 / 2)\end{array}$ \\
\hline $\begin{array}{l}{ }^{6} \mathrm{P}_{5 / 2}+ \\
{ }^{4} \mathrm{P}_{3 / 2}\end{array}$ & $\begin{array}{l}27360 \\
27427\end{array}$ & $\begin{array}{r}1 \\
0.82\end{array}$ & $\begin{array}{l}27390.8 \\
27445.6 \\
27486.9 \\
27506.6 \\
27574.7 \\
\end{array}$ & $\begin{array}{l}27385.2 \\
27428.9 \\
27443.7 \\
27477.7 \\
27520.2\end{array}$ & $\begin{array}{l}21 * 4 \mathrm{P} 2(3 / 2)+19 * 6 \mathrm{P}(3 / 2)+8 * 4 \mathrm{D} 2(3 / 2)+7 * 4 \mathrm{P} 1(3 / 2) \\
59 * 6 \mathrm{P}(5 / 2)+22 * 4 \mathrm{P} 2(5 / 2) \\
33 * 6 \mathrm{P}(5 / 2)+13 * 4 \mathrm{P} 2(5 / 2)+10 * 4 \mathrm{P} 2(3 / 2)+10 * 6 \mathrm{P}(3 / 2) \\
29 * 6 \mathrm{P}(5 / 2)+12 * 4 \mathrm{P} 2(3 / 2)+11 * 6 \mathrm{P}(3 / 2)+11 * 4 \mathrm{P} 2(5 / 2) \\
61 * 6 \mathrm{P}(5 / 2)+24 * 4 \mathrm{P} 2(5 / 2)\end{array}$ \\
\hline${ }^{4} I_{11 / 2}$ & & & $\begin{array}{l}27852.1 \\
27902.2 \\
27953.2 \\
28028.9 \\
28054.1 \\
28120.4\end{array}$ & $\begin{array}{l}27851.3 \\
27889.9 \\
27931.2 \\
28001.3 \\
28040.1 \\
28077.5\end{array}$ & $\begin{array}{l}59 * 4 I 3(11 / 2)+10 * 4 K 1(11 / 2)+9 * 4 I 1(11 / 2) \\
59 * 4 I 3(11 / 2)+11 * 4 K 1(11 / 2)+9 * 4 I 1(11 / 2) \\
59 * 4 I 3(11 / 2)+11 * 4 K 1(11 / 2)+8 * 4 I 1(11 / 2) \\
61 * 4 I 3(11 / 2)+10 * 4 K 1(11 / 2)+9 * 4 I 1(11 / 2) \\
62 * 4 I 3(11 / 2)+10 * 4 K 1(11 / 2)+9 * 4 I 1(11 / 2) \\
62 * 4 I 3(11 / 2)+10 * 4 K 1(11 / 2)+9 * 4 I 1(11 / 2)\end{array}$ \\
\hline $\begin{array}{l}{ }^{6} \mathrm{P}_{7 / 2}+ \\
{ }^{4} \mathrm{M}_{15 / 2}\end{array}$ & $\begin{array}{l}28433 \\
28506\end{array}$ & $\begin{array}{l}-0.91 \\
-0.81\end{array}$ & $\begin{array}{l}28371.1 \\
28449.5 \\
28462.3 \\
28488.8 \\
28519.6 \\
28541.5 \\
28571.3 \\
28592.3 \\
28619.1 \\
28771.8 \\
28851.4 \\
28893.5\end{array}$ & $\begin{array}{l}28360.0 \\
28413.2 \\
28446.6 \\
28457.0 \\
28491.4 \\
28519.1 \\
28527.5 \\
28550.8 \\
28624.2 \\
28731.9 \\
28771.7 \\
28844.2\end{array}$ & $\begin{array}{l}48 * 4 M(15 / 2)+14 * 4 I 3(15 / 2)+11 * 4 L(15 / 2) \\
40 * 4 M(15 / 2)+12 * 4 I 3(15 / 2)+12 * 6 \mathrm{P}(7 / 2)+9 * 4 \mathrm{~L}(15 / 2) \\
64 * 6 \mathrm{P}(7 / 2)+6 * 4 \mathrm{M}(15 / 2)+5 * 4 \mathrm{~F}(7 / 2) \\
13 * 4 \mathrm{I}(15 / 2)+10 * 4 \mathrm{~L}(15 / 2)+45 * 4 \mathrm{M}(15 / 2)+5 * 6 \mathrm{P}(7 / 2) \\
60 * 6 \mathrm{P}(7 / 2)+8 * 4 \mathrm{M}(15 / 2) \\
12 * 4 I 3(15 / 2)+40 * 4 \mathrm{M}(15 / 2)+11 * 6 \mathrm{P}(7 / 2)+9 * 4 \mathrm{~L}(15 / 2) \\
62 * 6 \mathrm{P}(7 / 2)+7 * 4 \mathrm{M}(15 / 2) \\
52 * 6 \mathrm{P}(7 / 2)+14 * 4 \mathrm{M}(15 / 2) \\
33 * 4 \mathrm{M}(15 / 2)+22 * 6 \mathrm{P}(7 / 2)+10 * 4 I 3(15 / 2)+8 * 4 \mathrm{~L}(15 / 2) \\
45 * 4 \mathrm{M}(15 / 2)+14 * 4 \mathrm{I}(15 / 2)+13 * 4 \mathrm{~L}(15 / 2) \\
45 * 4 \mathrm{M}(15 / 2)+14 * 433(15 / 2)+13 * 4 \mathrm{~L}(15 / 2) \\
45 * 4 \mathrm{M}(15 / 2)+14 * 4 \mathrm{I}(15 / 2)+13 * 4 \mathrm{~L}(15 / 2)\end{array}$ \\
\hline
\end{tabular}


Table S4. The free ion parameters used in the energy calculations given in Tables S2 and S3. The same free ion parameters are used for each metal site in the $\left[\operatorname{Ln}_{2} \mathrm{~L}(\mathrm{OAC})_{4}\right]^{+}$dimer.

\begin{tabular}{|l|l|l|}
\hline Parameter & Nd [17] & Dy [18] \\
\hline \hline$F^{2}$ & 71934 & 90840 \\
\hline$F^{4}$ & 52276 & 63956 \\
\hline$F^{6}$ & 35466 & 48775 \\
\hline$\zeta$ & 879.1 & 1921.0 \\
\hline$\alpha$ & 22.14 & 16.803 \\
\hline$b$ & -653.5 & -595.0 \\
\hline$\gamma$ & 1565.1 & 1882.0 \\
\hline$M^{0}$ & 1.9420 & 2.60 \\
\hline$P^{2}$ & 281.00 & 551.0 \\
\hline$T^{2}$ & 354.7 & 283.0 \\
\hline$T^{3}$ & 40.2 & 26.4 \\
\hline$T^{4}$ & 59.4 & -10.3 \\
\hline$T^{6}$ & -293.4 & -349.0 \\
\hline$T^{7}$ & 348.5 & 387.0 \\
\hline$T^{8}$ & 348.8 & 338.0 \\
\hline$M^{0}: M^{2}: M^{4}$ in ratio 1.00:0.56:0.38; $P^{2}: P^{4}: P^{6}$ in ratio $1.00: 0.75: 0.50$
\end{tabular}

The $M^{n}$ parameters are used for both spin-other-orbit and spin-spin interactions [19]. 
Table S5. The ligand field of sites $\operatorname{Ln} 1$ and $\operatorname{Ln} 2$. The AOM parameters $e_{\sigma}$ and $e_{\pi}$ are determined by the bond lengths (see text). The angles $\theta, \varphi$ are from the crystal structure with respect to a molecular coordinate system defined as the $z$ axis along the $\operatorname{Ln} 1-\operatorname{Ln} 2$ direction and the $y$ axis perpendicular to the Ln1-Ln2-O9PH plane.

\begin{tabular}{|l|r|r|r|r|}
\hline \multicolumn{1}{|c|}{$\operatorname{Ln} 1$} & $\mathrm{e}_{\sigma}\left[\mathrm{cm}^{-1}\right]$ & $\mathrm{e}_{\pi}\left[\mathrm{cm}^{-1}\right]$ & \multicolumn{1}{c|}{$\theta\left[^{\circ}\right]$} & \multicolumn{1}{c|}{$\varphi\left[^{\circ}\right]$} \\
\hline \hline O9PH & 187.0 & 138.0 & 36.02 & 0.00 \\
\hline O1AC & 196.0 & 145.0 & 173.02 & 169.46 \\
\hline O2AC & 146.0 & 108.0 & 118.26 & 177.59 \\
\hline O3AC & 196.0 & 145.0 & 72.23 & 248.90 \\
\hline O5AC & 194.0 & 143.0 & 38.62 & 161.58 \\
\hline N1 & 653.0 & 0.0 & 108.94 & 31.38 \\
\hline N1PY & 619.0 & 101.0 & 94.28 & 96.89 \\
\hline N2PY & 642.0 & 106.0 & 106.83 & 319.10 \\
\hline \hline Ln2 & & & & \\
\hline O9PH & 161.0 & 119.0 & 35.25 & 0.00 \\
\hline O4AC & 163.0 & 120.0 & 68.51 & 109.79 \\
\hline O5AC & 115.0 & 84.0 & 35.78 & 198.42 \\
\hline O6AC & 108.0 & 80.0 & 87.19 & 184.09 \\
\hline O7AC & 133.0 & 98.0 & 81.90 & 261.52 \\
\hline O8AC & 135.0 & 99.0 & 134.47 & 246.86 \\
\hline N2 & 594.0 & 0.0 & 103.55 & 44.19 \\
\hline N3PY & 611.0 & 101.0 & 109.11 & 335.06 \\
\hline N4PY & 594.0 & 98.0 & 147.31 & 107.86 \\
\hline
\end{tabular}


Table S6. The equivalent ligand field to the AOM parameters in Table S4 in terms of the $B_{k q}$ parameters (all values in $\mathrm{cm}^{-1}$ ). The $B_{k q}$ parameters depend on the orientation of the coordinate system. A different orientation of the molecular axes will give in different values of $B_{k q}$ but will result in the same calculated values as given in Tables S1 and S2.

\begin{tabular}{|l||c|c|r|c|}
\hline \multicolumn{1}{|c||}{} & \multicolumn{2}{c|}{ Ln1 } & \multicolumn{2}{c|}{ Ln2 } \\
\hline \hline & $B_{k q}$ & \multicolumn{1}{c|}{$B_{k q}^{\prime}$} & $B_{k q}$ & $B_{k q}^{\prime}$ \\
\hline \hline$B_{20}$ & -241.1112 & - & -59.3107 & - \\
\hline$B_{21}$ & -174.5708 & -69.7026 & -137.1880 & -131.7868 \\
\hline$B_{22}$ & -62.3106 & -111.6471 & 40.2660 & -3.7924 \\
\hline$B_{40}$ & 504.6157 & - & 32.1808 & - \\
\hline$B_{41}$ & 581.7992 & 193.2732 & 702.9301 & -389.2490 \\
\hline$B_{42}$ & 605.3320 & 7.6392 & -221.7425 & -231.0015 \\
\hline$B_{43}$ & 413.1949 & 133.2036 & 11.6428 & 154.8686 \\
\hline$B_{44}$ & -74.6690 & 208.3797 & -191.9966 & -238.6755 \\
\hline$B_{60}$ & 50.7495 & - & -167.0971 & - \\
\hline$B_{61}$ & -489.0192 & -103.6873 & -438.0002 & -184.1288 \\
\hline$B_{62}$ & -355.0176 & -141.0919 & -413.7643 & -89.8566 \\
\hline$B_{63}$ & -141.1603 & 15.1552 & -437.6837 & 215.1074 \\
\hline$B_{64}$ & -284.3568 & -68.0181 & 186.2086 & 109.1304 \\
\hline$B_{65}$ & 822.3279 & -427.6836 & 539.7832 & 537.1132 \\
\hline$B_{66}$ & -864.1667 & -38.0688 & -292.0057 & -602.1191 \\
\hline
\end{tabular}


Table S7. The calculated g-values of the ${ }^{4} I_{9 / 2}$ and ${ }^{6} \mathrm{H}_{15 / 2}$ ground state multiplets in the $\mathrm{Nd}_{2} \mathrm{~L}$ and $\mathrm{Dy}{ }_{2} \mathrm{~L}$ dimers respectively.

\begin{tabular}{|c|c|c|c|c|c|c|c|}
\hline Nd1 & & & & $\mathrm{Nd} 2$ & & & \\
\hline Eng & $\left|\mathrm{g}_{1}\right|$ & $\left|\mathrm{g}_{2}\right|$ & $\left|\mathrm{g}_{3}\right|$ & \multicolumn{1}{|c|}{ Eng } & $\left|\mathrm{g}_{1}\right|$ & $\left|\mathrm{g}_{2}\right|$ & $\left|\mathrm{g}_{3}\right|$ \\
\hline 0.0 & 0.405 & 0.977 & 4.958 & 0.0 & 0.006 & 1.618 & 3.561 \\
\hline 78.0 & 0.038 & 1.620 & 3.299 & 58.5 & 0.223 & 1.024 & 2.309 \\
\hline 150.2 & 0.404 & 0.985 & 3.318 & 103.7 & 0.444 & 1.682 & 3.441 \\
\hline 391.9 & 0.967 & 1.320 & 2.460 & 313.8 & 1.225 & 1.822 & 3.126 \\
\hline 526.5 & 0.412 & 1.244 & 3.189 & 484.2 & 1.535 & 2.321 & 3.099 \\
\hline & & & & & & & \\
\hline Dy1 & & & & Dy2 & & & \\
\hline Eng & $\left|\mathrm{g}_{1}\right|$ & $\left|\mathrm{g}_{2}\right|$ & $\left|\mathrm{g}_{3}\right|$ & Eng & $\left|\mathrm{g}_{1}\right|$ & $\left|\mathrm{g}_{2}\right|$ & $\left|\mathrm{g}_{3}\right|$ \\
\hline 0.0 & 0.344 & 0.498 & 19.348 & 0.0 & 0.071 & 2.407 & 15.739 \\
\hline 38.8 & 0.287 & 0.421 & 17.634 & 43.7 & 0.254 & 1.153 & 12.113 \\
\hline 105.2 & 0.753 & 7.303 & 11.745 & 81.5 & 3.239 & 4.288 & 9.241 \\
\hline 148.9 & 0.700 & 6.808 & 9.319 & 135.2 & 1.200 & 2.829 & 10.273 \\
\hline 265.2 & 1.985 & 5.733 & 11.845 & 213.8 & 1.316 & 3.426 & 14.482 \\
\hline 325.2 & 2.221 & 6.532 & 9.520 & 309.5 & 0.025 & 5.240 & 10.381 \\
\hline 416.8 & 0.293 & 2.250 & 16.399 & 362.3 & 2.869 & 4.658 & 10.886 \\
\hline 498.2 & 0.978 & 1.256 & 18.734 & 431.9 & 0.123 & 0.898 & 16.752 \\
\hline & & & & & & & \\
\hline
\end{tabular}


Table S8. Crystal-field parameters for the description of the splitting of the ground-state multiplet $J=15 / 2$ given in $\mathrm{cm}^{-1}$. The Parameters have been calculated by decoupling of the wavefunction in terms of Extended Stevens operators.

\begin{tabular}{|c|c|c|c|c|c|}
\hline \multicolumn{3}{|l|}{ Dy1 } & \multicolumn{3}{|l|}{ Dy2 } \\
\hline k & $q$ & $B^{q}{ }_{k}$ & $k$ & $q$ & $B_{k}^{q}$ \\
\hline 2 & -2 & 0.7328 & 2 & -2 & -0.1831 \\
\hline 2 & -1 & 0.2712 & 2 & -1 & -0.8008 \\
\hline 2 & 0 & -0.9308 & 2 & 0 & -1.2439 \\
\hline 2 & 1 & -3.1739 & 2 & 1 & -1.4246 \\
\hline 2 & 2 & 2.4380 & 2 & 2 & -0.3916 \\
\hline 4 & -4 & 0.0084 & 4 & -4 & -0.0084 \\
\hline 4 & -3 & 0.0018 & 4 & -3 & -0.0134 \\
\hline 4 & -2 & -0.0089 & 4 & -2 & -0.0034 \\
\hline 4 & -1 & -0.0068 & 4 & -1 & -0.0100 \\
\hline 4 & 0 & -0.0058 & 4 & 0 & -0.0003 \\
\hline 4 & 1 & 0.0097 & 4 & 1 & 0.0058 \\
\hline 4 & 2 & 0.0116 & 4 & 2 & -0.0031 \\
\hline 4 & 3 & -0.0358 & 4 & 3 & -0.0409 \\
\hline 4 & 4 & 0.0137 & 4 & 4 & 0.0054 \\
\hline 6 & -6 & -0.0001 & 6 & -6 & 0.0003 \\
\hline 6 & -5 & -0.0002 & 6 & -5 & -0.0001 \\
\hline 6 & -4 & 0.0000 & 6 & -4 & 0.0001 \\
\hline 6 & -3 & 0.0001 & 6 & -3 & 0.0002 \\
\hline 6 & -2 & 0.0000 & 6 & -2 & 0.0001 \\
\hline 6 & -1 & 0.0001 & 6 & -1 & 0.0002 \\
\hline 6 & 0 & 0.0000 & 6 & 0 & 0.0000 \\
\hline 6 & 1 & 0.0001 & 6 & 1 & 0.0000 \\
\hline 6 & 2 & -0.0002 & 6 & 2 & 0.0000 \\
\hline 6 & 3 & -0.0002 & 6 & 3 & 0.0001 \\
\hline 6 & 4 & 0.0001 & 6 & 4 & 0.0001 \\
\hline 6 & 5 & -0.0009 & 6 & 5 & 0.0004 \\
\hline 6 & 6 & 0.0001 & 6 & 6 & 0.0001 \\
\hline
\end{tabular}

$97.39 \%$

Extended Stevens operators (ESO) of the ground multiplet calculated by the SINGLE_ANISO program. ${ }^{25,44}$ This is the decoupling of the calculated wavefunction in terms of ESO. Crystal-Field Hamiltonian:

$$
H_{C F}=\sum_{k=2,4,6} \sum_{q=-k, k}\left[B_{k}^{q} O_{k}^{q}\right]
$$

The orientation of the ab initio calculated main magnetic axes $\left(g_{z}\right)$ of the Kramer doublets on the magnetic centers Dy1 and Dy2 is visualized in Figure S10. 
Table S9. Given are the total coefficients of the M, projections of the RASSI coupled wavefunction.

\begin{tabular}{l|ll}
$\begin{array}{l}\text { Kramers- } \\
\text { Doublet }\end{array}$ & Dy1 & Dy2 \\
\hline 1 & $0.87 \mid \pm 15 / 2>$ & $0.94 \mid \pm 15 / 2>$ \\
2 & $0.32| \pm 5 / 2>; 0.30| \pm 3 / 2>; 0.27 \mid \pm 1 / 2>$ & $0.42| \pm 13 / 2>; 0.30| \pm 11 / 2>; 0.19 \mid \pm 9 / 2>$ \\
3 & $0.27| \pm 13 / 2>; 0.26| \pm 11 / 2>$ & $0.22| \pm 7 / 2>; 0.21| \pm 13 / 2>; 0.18 \mid \pm 9 / 2>$ \\
4 & $0.23| \pm 3 / 2>; 0.18| \pm 1 / 2>; 0.18 \mid \pm 13 / 2>$ & $0.28| \pm 5 / 2>; 0.24| \pm 3 / 2>$ \\
5 & $0.31| \pm 7 / 2>; 0.21| \pm 11 / 2>$ & $0.46| \pm 7 / 2>; 0.26| \pm 1 / 2>$ \\
6 & $0.27| \pm 3 / 2>; 0.26| \pm 9 / 2>; 0.25 \mid \pm 1 / 2>$ & $0.35| \pm 3 / 2>; 0.22| \pm 5 / 2>$ \\
7 & $0.23| \pm 9 / 2>; 0.22| \pm 3 / 2>; 0.2 \mid \pm 1 / 2>$ & $0.42| \pm 1 / 2>; 0.32| \pm 3 / 2>$ \\
8 & $0.42| \pm 9 / 2>; 0.38| \pm 7 / 2>; 0.36 \mid \pm 11 / 2>$ & $0.36| \pm 5 / 2>; 0.30| \pm 1 / 2>$
\end{tabular}



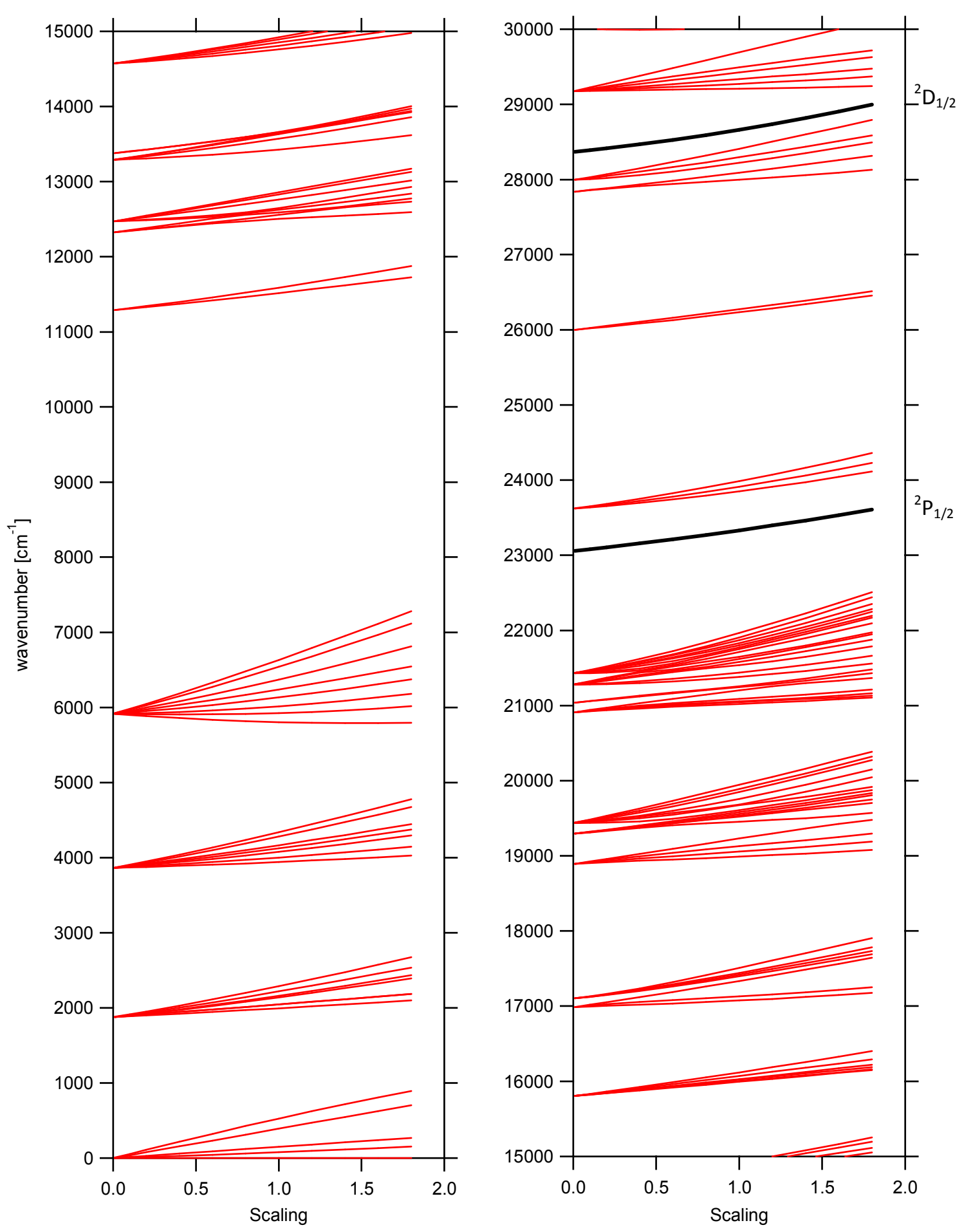

Figure 59. The calculated levels of the $\mathrm{Nd} 1$ center of $\left[\mathrm{Nd}_{2} \mathrm{~L}(\mathrm{OAc})_{4}\right]^{+}$as a function of ligand field strength (The $e_{\sigma}$ and $e_{\pi} \mathrm{Nd} 1$ parameters multiplied by the scaling factor shown). 

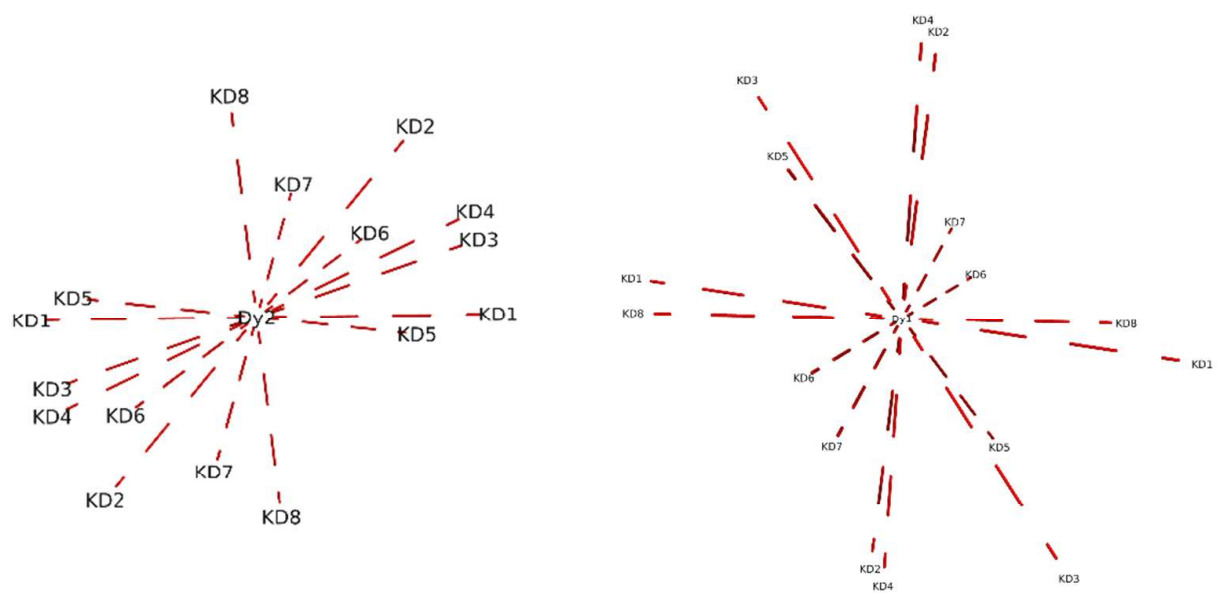

Figure S10. Orientation of the ab initio calculated main magnetic axes $\left(g_{z}\right)$ of the Kramer Doublets on the magnetic centers Dy1 and Dy2.

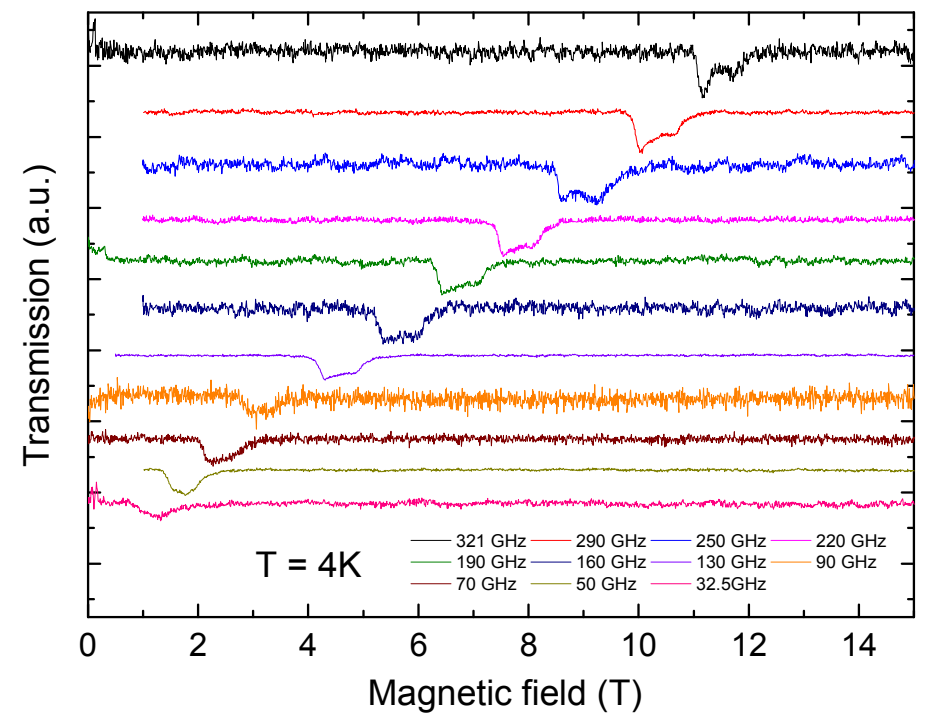

Figure S11. Frequency dependence of the HF-EPR spectra of $\mathrm{Gd}_{2}$ at $4 \mathrm{~K}$. Spectra are shifted for visibility. 


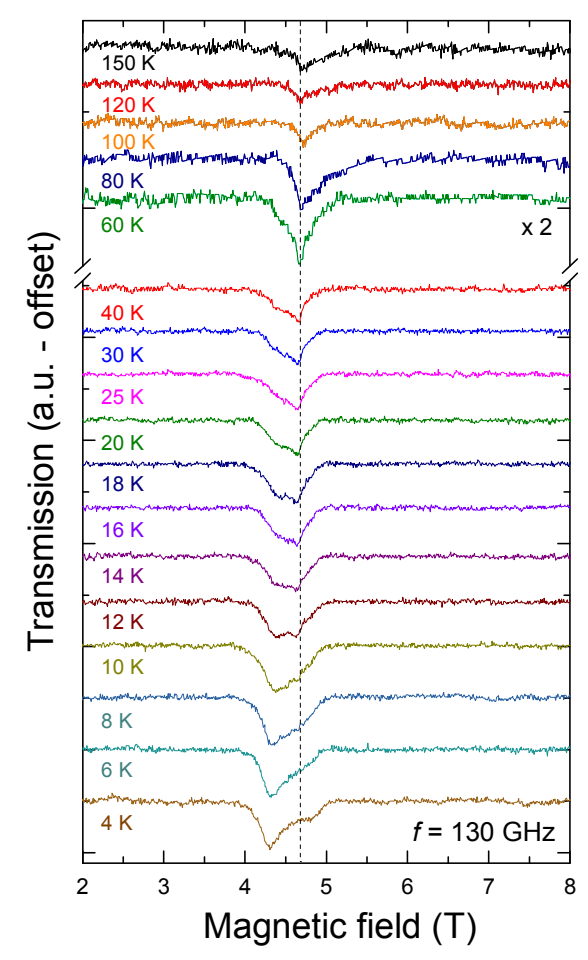

Figure S12. Temperature dependence of the HF-EPR spectra of $\mathrm{Gd}_{2}$ at $130 \mathrm{GHz}$. The vertical dashed line indicates the field position corresponding to $g=1.98$. Spectra are shifted for visibility and multiplied by 2 for $T \geq 60 \mathrm{~K}$.

\section{References}

(1) SAINT Bruker AXS GmbH, Karlsruhe, Germany: 1997-2013.

(2) CrysAlisPro Agilent Technologies, UK Ltd., Oxford: 2011-2014.

(3) CrysAlisPro SCALE3 ABSPACK CrysAlisPro, SCALE3 ABSPACK CrysAlisPro; Agilent Technologies UK Ltd.: Oxford, 2011-2014.

(4) Blessing, R. H., Acta Cryst. 1995, A51, 33-38.

(5) Sheldrick, G. M. SADABS, Bruker AXS: Karlsruhe, Germany, 2004-2014.

(6) Palatinus, L. SUPERFLIP; EPF Lausanne: Switzerland, 2007.

(7) Sheldrick, G. M. SHELXL-20xx; University of Göttingen and Bruker AXS GmbH, Karlsruhe, Germany: 2012-2014.

(8) Sheldrick, G. M., Acta Cryst. 2008, A64, 112-122.

(9) Sheldrick, G. M., Acta Cryst. 2015, C71, 3.

(10) Sluis, P. v. d.; Spek, A. L., Acta Cryst. 1990, A46, 194-201.

(11) Spek, A. L. PLATON, PLATON, Utrecht University, The Netherlands: 2003.

(12) Spek, A. L., J. Appl. Cryst. 2003, 36, 7-13. 
(13) Urland, W., Personal Communication.

(14) Schäffer, C. E. Structure \& Bonding 1968, 5, 68-95.

(15) Flanagan, B. M.; Bernhardt, P. V.; Krausz, E. R.; Lüthi, S. R.; Riley, M. J. Inorg. Chem. 2002, 41, 5024-5033.

(16) Urland, W. Chem. Phys. 1976, 14, 393-401.

(17) Yeung, Y. Y.; Tanner, P. A.; J. Alloys Comp. 2013, 575, 54-60.

(18) Rana, R. S.; Shertzer, J.; Kaseta, F. W.; Garvey, R.; Rana, D.; Feng, S. Y.; J. Chem. Phys., 1988, $88,2242-2248$.

(19) Judd, B. R.; Crosswhite, H. M.; Crosswhite, H.; Phys. Rev. 1968, 169, 130-138. 